Wilfrid Laurier University

Scholars Commons @ Laurier

Physics and Computer Science Faculty

Publications

Physics and Computer Science

2011

\title{
Ion-Exchanged Glass Waveguide Technology: A Review
}

Ari Tervonen

Aalto University

Brian R. West

Wilfrid Laurier University, bwest@wlu.ca

Seppo Honkanen

Aalto University

Follow this and additional works at: https://scholars.wlu.ca/phys_faculty

\section{Recommended Citation}

Tervonen, Ari; West, Brian R.; and Honkanen, Seppo, "Ion-Exchanged Glass Waveguide Technology: A Review" (2011). Physics and Computer Science Faculty Publications. 91.

https://scholars.wlu.ca/phys_faculty/91

This Article is brought to you for free and open access by the Physics and Computer Science at Scholars Commons @ Laurier. It has been accepted for inclusion in Physics and Computer Science Faculty Publications by an authorized administrator of Scholars Commons @ Laurier. For more information, please contact scholarscommons@wlu.ca. 


\section{lon-exchanged glass waveguide technology: a review}

Ari Tervonen

Aalto University

School of Electrical Engineering

Department of Micro and Nanosciences

P.O. Box 13500, FI-00076 Aalto

Espoo, Finland

E-mail: ari.tervonen@tkk.fi

Brian R. West

Wilfrid Laurier University

Department of Physics \& Computer Science

Waterloo, Ontario N2L 3C5

Canada

Seppo Honkanen

Aalto University

School of Electrical Engineering

Department of Micro and Nanosciences

P.O. Box 13500, FI-00076 Aalto

Espoo, Finland

\begin{abstract}
We review the history and current status of ion exchanged glass waveguide technology. The background of ion exchange in glass and key developments in the first years of research are briefly described. An overview of fabrication, characterization and modeling of waveguides is given and the most important waveguide devices and their applications are discussed. Ion exchanged waveguide technology has served as an available platform for studies of general waveguide properties, integrated optics structures and devices, as well as applications. It is also a commercial fabrication technology for both passive and active waveguide components. (C) 2011 Society of Photo-Optical Instrumentation Engineers (SPIE). [DOI: 10.1117/1.3559213]
\end{abstract}

Subject terms: ion exchange; waveguides; integrated optics.

Paper 100979SSR received Nov. 24, 2010; revised manuscript received Jan. 21, 2011; accepted for publication Jan. 31, 2011; published online Mar. 29, 2011.

\section{Introduction}

Ion exchange in glass ${ }^{1,2}$ is a widely applied technology for optical waveguide fabrication. By local substitution of relatively mobile ions (typically $\mathrm{Na}^{+}$) originally in glass by other ions with different size and polarizability, a change of refractive index in selected regions is achieved, allowing patterning of waveguiding regions into planar substrates. Several reviews ${ }^{3-9}$ on ion-exchanged waveguide technology and thousands of research papers connected to it have been published in the past 40 years during which this technology has been studied.

Ion exchange in glass has an extensive history. ${ }^{10}$ It has been used for centuries to modify optical absorption properties of glass for purposes of decoration and coloring. ${ }^{11,12}$ Glass luster on ceramics with metallic nanoparticles from ion exchange is known from the early Islamic culture during the 10th century. ${ }^{13}$ Glass strengthening by surface ion exchange ${ }^{14-16}$ was the first extensive industrial application of ion exchange in glass.

Shortly after the introduction of the concept of integrated optics, ion exchange in glass was proposed as a waveguide fabrication process, ${ }^{17,18}$ based on previous use in glass rod lenses and fibers. ${ }^{19}$ In fact, development of ion-exchanged waveguide technology closely parallels the emergence of practical graded-index optics. $^{20}$

Glass is of course a well-known optical material and glass waveguides have several advantages including low material cost, compatibility with optical fibers, low propagation loss and birefringence, and high stability and reliability. Ion exchange as a fabrication process promises simplicity and economy, not requiring complicated manufacturing equipment. It allows for batch processing, and also flexibility of process and glass choices, so it can be adapted for many applications. Due to their availability, ion-exchanged waveguides are

0091-3286/2011/\$25.00 @ 2011 SPIE widely used for diverse studies, research tasks, experiments and prototyping work. Ion exchange technology also has proven applicability for industrial manufacturing of waveguide components; however, there are then special demands on fabrication to meet requirements for components to be applied in field.

There has been no single defined target for research on ion-exchanged waveguides. Though much of the work has concentrated on developing fabrication technology for particular waveguide device applications, much of it has been more general studies in the field of integrated optics, with ion exchange only used as an available technology platform.

\section{Early Years of Ion-Exchanged Waveguides}

The first published work on ion-exchanged waveguides appeared in 1972, authored by Izawa and Nakagome. ${ }^{21}$ The waveguides described therein used $\mathrm{Tl}^{+}$ion exchange from a molten salt bath for $\mathrm{Na}^{+}$and $\mathrm{K}^{+}$ions in glass, and already introduced two key processing techniques: field-assisted ion exchange to achieve migration of ions deeper into glass and a second step introducing original ions back into the glass to bury the waveguide under the surface, achieving low loss. In addition to planar waveguides, there were demonstrated multimode channel waveguides fabricated using a lithographically patterned mask film. Though remarkably advanced work, it was still mainly a basic demonstration of feasibility. Much more thorough work would be needed to realize the potential of ion-exchanged waveguides and to establish the capacity for glass integrated optics fabrication. The following year, a publication by Giallorenzi et al. studied planar waveguide fabrication by purely thermal diffusion. ${ }^{22}$ Different exchanged ions were compared: silver, potassium, and thallium. Planar waveguide properties such as concentration and refractive index profiles and losses were studied. In particular, an order of magnitude lower refractive index increase was found to be produced by potassium exchange (0.008) in 
comparison to silver exchange (0.08). Following these first studies, Saruwatari and Izawa demonstrated in 1974 the first nonfiber glass waveguide laser using their Tl-exchange process in Nd-doped borosilicate glass. This was a 4-mm long multimode waveguide in which pulsed laser operation was achieved. ${ }^{23}$ This early waveguide laser work was largely forgotten, and it was more than a decade before research on ion-exchanged waveguides in rare-earth doped glasses really started.

Some additional experimental work on thermal silverexchanged planar waveguides followed, with characterization of index profiles and basic mode properties. Gedeon and Carnstam fabricated multimode strip waveguides using such a process and characterized their bending losses. ${ }^{24}$ The first single-mode channel waveguides-also produced by silver ion exchange-were demonstrated by Gallagher and de la Rue in $1976 .^{25}$

At the time, much emphasis was placed on the fabrication of multimode waveguides. This was partially due to technological challenges encountered in narrow-line lithography, particularly as most of the work was done with visible wavelengths and with a relatively large refractive index contrast. However, there was also a need for components to be used in multimode fiber communication. Some new fabrication processes were targeting multimode waveguide fabrication, such as lithium ion exchange. ${ }^{26}$ Also, the first work on silver-exchanged waveguides using solid-state Ag film sources with field-assisted process ${ }^{27}$ in particular, provided the possibility of achieving highly multimode waveguides. Nearly circular strip waveguides were demonstrated by Viljanen and Leppihalme using such a "dry" process along with thermal annealing. ${ }^{28}$ Targeting multimode waveguides also brought added interest in field-assisted fabrication processes, which could provide almost step-index profiles. Subsequent work by Lilienhof et al. showed that profile shapes were primarily determined by the electric field distribution in glass. ${ }^{29}$ Searching for alternate processes, the exchange of $\mathrm{Cs}^{+}$and $\mathrm{Rb}^{+}$ions was studied, and resulting steep profiles were explained by the mixed alkali effect in glasses. ${ }^{30}$

Detailed studies on silver ion exchange in planar waveguides followed, seeking to obtain better knowledge and understanding of the exchange process, which was needed for controlled fabrication and to establish the repeatability of manufacturing. Stewart et al. performed a study of Ag-diffused waveguide characteristics, determining the relation of diffusion profiles and mode properties to process parameters. ${ }^{31}$ Millar and Hutchins evaluated manufacturing tolerances of such waveguides. ${ }^{32}$ Stewart and Laybourn studied the use of dilute silver nitrate melts, showing that this provided advantages in process control, ${ }^{33}$ as had been earlier suggested. ${ }^{34}$ Based on regular solution theory, they determined the relationship between the waveguide surface index and silver concentration in the salt melt. This added significantly to the flexibility of silver exchange processes, since refractive index contrast could be selected for a given application.

Interestingly, the first functional device fabricated by ion exchange in glass was a quite advanced active $\mathrm{Nd}$-doped waveguide laser ${ }^{23}$ discussed in Sec. 6.3. Several basic passive devices realized with ion-exchanged waveguides were also demonstrated. A guided-wave polarizer was made with planar waveguides, having a section with smaller depth, below the cutoff for fundamental TM mode. ${ }^{35}$ A taper to optimize coupling of a single-mode strip waveguide to singlemode fiber achieved the required diffusion profile by dipping the masked substrate slowly into the salt melt during the process. ${ }^{36}$ A five-channel wavelength multiplexer was demonstrated, combining a chirped grating with multimode silver-exchanged waveguides. ${ }^{37}$ Separate from the emphasis on silver exchange, single-mode 1-to-N star couplers were demonstrated with potassium exchange. ${ }^{38} \mathrm{~K}^{+}$ion exchange was also used to make the first thermo-optic waveguide switches that combined miniature thin-film heaters deposited on waveguide structures, achieving millisecond switching with $0.5 \mathrm{~W}$ switching power. ${ }^{39}$

Development of ion-exchanged waveguides to provide a technology basis for the fabrication of guided-wave devices culminated in several papers by a group at University of Glasgow. With a goal of producing miniature optical ring resonators, they had developed a fabrication process for narrow, high-refractive-index-contrast silver-exchanged waveguides operating at $633 \mathrm{~nm}$ wavelength. ${ }^{40}$ Optimized directional coupler and bend structures could be made with these waveguides, ${ }^{41}$ and ring resonators with 0.5 -mm curve radius were demonstrated based on such structures, with finesse of 15 and $0.7-\mathrm{dB}$ round-trip loss. ${ }^{42}$ A related work was a study on a single-mode waveguide Y-junction made using the same process. ${ }^{43}$ A contemporary example of a single-mode device based on potassium-exchanged waveguides was a directional coupler power divider, in which dimensional requirements were considerably relaxed by a two-step process; this device was demonstrated at visible wavelengths. ${ }^{44}$

After more than a decade of research in ion-exchanged waveguides, interest had gradually grown, and basic demonstrations of the feasibility of single-mode devices and of flexibility of the process had built the foundation for further prospects of this technology, including serious possibilities for commercial production. The status of ion-exchanged glass waveguide technology at the time was reviewed by Findakly. $^{3}$

\section{Waveguide Fabrication Processes}

The ion exchange processes for waveguide fabrication can, in most cases, be represented as a sequence of between one and three process steps, configurations of which are shown in Fig. 1 (illustrated here for silver-sodium ion exchange). The first four configurations are used to introduce ions into glass. Figure 1(a) is the widely used thermal ion exchange from a molten salt source through a patterned mask. ${ }^{24}$ Figure 1(b) illustrates the field-assisted ion migration from a molten salt at the anode side. ${ }^{21}$ Figure 1 (c) is the field-assisted migration from a metal thin film source $;^{28}$ the thin film may be deposited on top of a patterned mask, or it can be directly patterned onto the glass surface. Figure 1(d) shows a field-assisted migration process, combining a patterned metal film with molten salt source at the anode surface; ${ }^{45,46}$ this can be used as a one-step process for producing buried waveguides, for after the metal film source has been consumed, this will directly continue as a field-assisted burial, similar to configuration [Fig. 1 $(\mathrm{g})]$.

Figures 1(e)-1(g) are used to modify the existing ion concentration established in glass in order to tailor the waveguide properties, so they are typically used as a second step in two-step fabrication processes. Figure 1(e) is a simple thermal annealing to redistribute the ions by diffusion inside the 


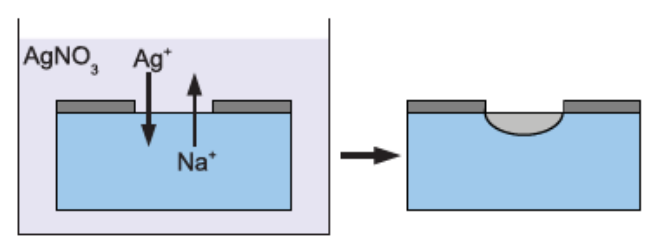

(a)

(b)

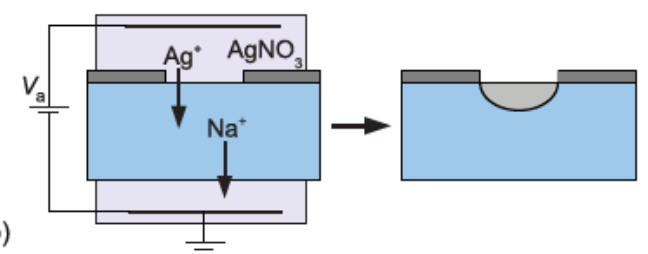

(c)

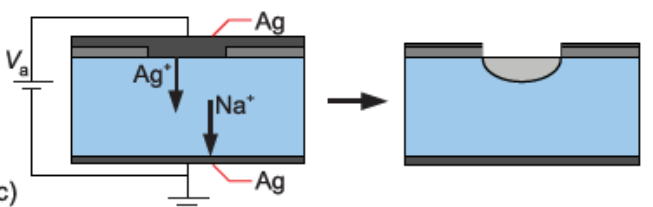

(d)
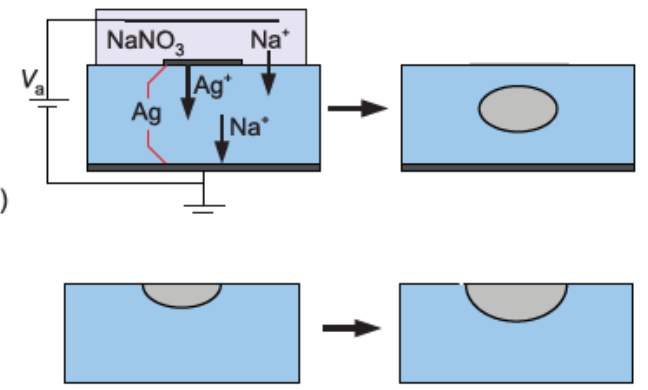

(e)

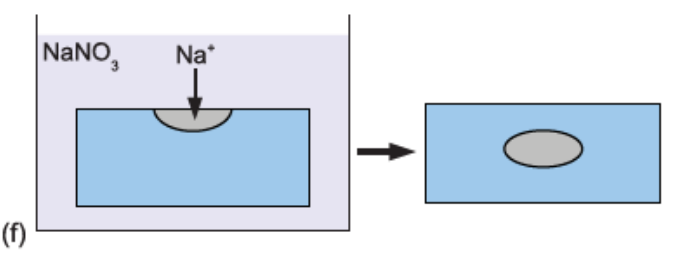

(g)

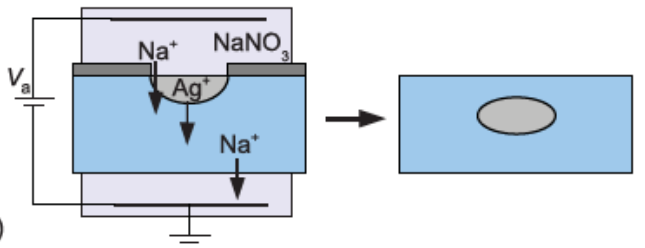

Fig. 1 lon exchange process configurations: (a) thermal exchange from molten salt; (b) field-assisted exchange from molten salt; (c) field-assisted exchange from a metal thin-film source; (d) singlestep field-assisted exchange and burial from a metal thin-film source with molten slat; $(e)$ thermal diffusion for a waveguide; $(f)$ thermal burial for a waveguide; and (g) field-assisted burial for a waveguide.

glass. ${ }^{28}$ In Fig. 1(f) a thermal burial is shown, in which ions originally in the glass are replaced back into the substrate at the surface, while the ions forming the waveguide are diffused deeper. ${ }^{47}$ Figure $1(\mathrm{~g})$ is the field-assisted burial step, ${ }^{21}$ which replaces the original ions back into glass at the surface, while the waveguide can migrate deeper into the substrate by the electric field.
For salt melt sources, nitrates with low melting temperature are typically used. The salt source can also have a diluted concentration of the index-increasing ion. Eutectic mixtures of salt can be used to reach even lower process temperatures. ${ }^{48}$

Laboratory equipment for processes based on thermal diffusion and exchange can be relatively simple: an oven with a suitable container for the salt melt. Accurate control of the process temperature is the key factor for reproducibility of the process. For field-assisted processes with molten salts, processing equipment is somewhat more complicated, as electrical contacts must be made to the anode and cathode sides of the substrate, and these need to be maintained in electrical isolation. The literature on waveguide fabrication gives many descriptions of process arrangements; a more detailed description of equipment for field-assisted ion exchange can be found in Ref. 49 .

For channel waveguide fabrication, various mask materials with lithographical patterning have been used. Aluminum $^{40,45,50}$ and titanium ${ }^{45}$ are the most widely used metallic masks. Often, dielectric masks are preferable to metal films, as Ag nanoparticles can form under the mask edges. ${ }^{40}$ Additionally, conducting masks can generate an electric field in the glass, affecting ion concentration distributions. ${ }^{40,50}$ Furthermore, a thin depletion layer forms under a metal mask in field-assisted processes, affecting the ion concentration in this region. ${ }^{51}$ For these reasons, dielectric mask materials are often preferred; for example, aluminum oxide, ${ }^{40}$ silicon dioxide, and silicon nitride ${ }^{52}$ have been used. As waveguides can be wider than the ion source width due to lateral diffusion, narrow lithography linewidths are needed for single-mode waveguides. In particular, for deeply buried single-mode waveguides, the linewidth may need to be 1 to $2 \mu \mathrm{m}$.

The primary interest for practical components is in buried waveguides having profiles matched with optical fibers. Buried waveguides are immune to surface defects and environmental disturbances, they can achieve the symmetry needed for low birefringence and efficient coupling to fibers, and they provide the required surface area at the waveguide edge facet for fixing the attached fibers. It should be pointed out that although waveguides matched with singlemode fibers do not need high refractive index contrast, concentration of exchanged ions decreases substantially by diffusion during the deep burial step, and thus it is necessary to obtain a relatively high index increase to start with. One particular property of waveguides buried in a field-assisted step needs to be mentioned here: the burial depth has been found to depend on the width of the waveguide due to different mobilities of the ions in electric field (planar waveguides are buried to a greater depth during the process than are single-mode waveguides). ${ }^{53-55}$ This needs to be considered in devices combining channel and planar waveguides.

Thallium ion exchange was used in the first publication in the field, and it was also used for the first industrial fabrication processes. Typically achieved by $\mathrm{Tl}^{+} / \mathrm{K}^{+}$exchange, it was developed for waveguide fabrication by Corning and first applied to multimode waveguides, ${ }^{56}$ but then product development concentrated on single-mode waveguide component products, mainly 1-to-N splitters. ${ }^{57}$ Nippon Sheet Glass developed an alternative fully thermal process for more shallowly buried waveguides. ${ }^{47}$ The advantage of thallium is 
that a rather large index increase can be obtained. The main reason for the rather limited use of thallium ion exchange, particularly in research work, is its high toxicity. Another process developed to target component manufacturing is cesium ion exchange. A special glass, BGG21, was developed for $\mathrm{Cs}^{+} / \mathrm{K}^{+}$exchange and used in a field-assisted burial process for single-mode waveguides. ${ }^{58}$

The two most widely used ions in ion-exchange glass waveguide research activities are $\mathrm{Ag}^{+}$and $\mathrm{K}^{+}$. Potassium ion exchange has the advantage that it can easily be used to produce low-loss surface waveguides and its low refractive index increase allows relaxed mask opening dimensions. However, relatively high stresses are generated in $\mathrm{K}^{+}$ -exchanged glass and the index increase is largely caused by stress-optic effects, ${ }^{59}$ thus making waveguides birefringent. ${ }^{60}$ Additionally, $\mathrm{K}^{+}$exchange is not well-suited for buried waveguide fabrication, due to the low index contrast and the large difference in mobility of $\mathrm{K}^{+}$and $\mathrm{Na}^{+}$ions.

Silver ion exchange is, in many respects, quite ideal for waveguide fabrication, which explains its wide popularity. It can achieve a relatively high index increase (which can be decreased by melt dilution to meet single-mode requirements) and the mobility ratio of $\mathrm{Ag}^{+}$and $\mathrm{Na}^{+}$ions is not very low in most glasses. The main disadvantage found throughout many studies of silver-exchanged waveguides is the tendency of silver ions to reduce into neutral atoms, subsequently aggregating into metallic clusters in glass and forming an absorbing colloid. This can cause high waveguide losses, particularly with higher concentrations of $\mathrm{Ag}^{+}$ions. Many approaches have been taken to decrease this loss, including the use of diluted silver concentrations and avoiding metallic mask films in fabrication processes. It was soon understood that glass composition played a key role in the reduction of silver to metallic form, and that in particular impurity elements $\mathrm{Fe}$, $\mathrm{As}$, and $\mathrm{Sb}$ residing in the glass structure as polyvalent ions should be avoided, as $\mathrm{Ag}^{+}$ions can capture electrons from them. Most research work has used some readily available commercial glasses. Careful selection led to use of highquality glasses such as Corning 0211 with reduced silver particle formation. However, it was discovered that an optimal glass for silver ion exchange would have a very special composition, not only avoiding such impurities. ${ }^{61}$ In conventional sodium-containing glasses, sodium ions are associated with nonbridging oxygen atoms in the glass network, and silver ions exchanged into glass can extract electrons from these nonbridging oxygen atoms for reduction into silver atoms. By adding aluminum oxide into glass, the number of nonbridging oxygen sites is decreased. With molar concentrations of $\mathrm{Al}_{2} \mathrm{O}_{3}$ and $\mathrm{Na}_{2} \mathrm{O}$ in glass at the same level, a glass network with a different structure is established, in which sodium ions are not associated with nonbridging oxygens. $\mathrm{Ag}^{+}$ions exchanged into such aluminosilicate glasses are quite stable. In addition, boron can have a similar role as aluminum in the glass structure. A special glass was developed for silver ion exchange-BGG315,62 - of aluminoborosilicate composition. With only sodium present as alkali species, it made the truly binary ion exchange simpler to manage. This glass also had a fraction of the oxygen replaced by fluorine, and thus reached a refractive index value at the level of silica to match optical fibers. IOT was launched as a company in integrated optics components, with their technology of single-mode device manufacturing based on this glass.
The main difference between small-volume laboratory research on ion-exchanged waveguides and industrial-scale production is the availability of special glass substrates developed for this purpose, and to some extent, fabrication equipment sufficient for industrial quality manufacturing. The latter is particularly relevant with respect to field-assisted processes with molten salts, which are complicated to operate conveniently and with controllability to achieve the required reproducibility. As fabrication of small batches of special glasses for research purposes cannot typically reach sufficient optical quality for the glass material, such work is generally limited to the use of commercially available substrates.

When silver ion-exchanged waveguides are fabricated from $\mathrm{Ag}$ metal film sources, the process has differences from processes based on ion exchange from molten salt sources. ${ }^{63}$ Thermal diffusion from Ag film into glass is slow, so the process needs to be field-assisted in order to produce substantial silver ion concentrations. This process is electrochemical; electrolytic anode reactions were studied and explained in detail by Kapila and Plawsky. ${ }^{64}$ This technique has also been used to fabricate single-mode waveguides, first by the single-step burial process, ${ }^{65}$ and later by combining the field-assisted migration first step with annealing to produce single-mode fiber-compatible waveguides in a completely dry solid-state process, ${ }^{66}$ which has also been used to demonstrate a number of guided-wave devices. ${ }^{67,68}$ To date, no commercial manufacturing has utilized dry silver ion exchange.

Combinations of different ion exchange processes in fabricating waveguide structures on the same substrate have also been studied. In particular, waveguides from potassium and silver ion exchange produce index contrasts that differ by about an order of magnitude in a given glass. Also, since the $\mathrm{K}^{+}$ions have much lower mobility at a given temperature than $\mathrm{Ag}^{+}$ions, potassium ion exchange can be carried out first at a higher temperature, and when subsequent silver ion exchange is carried out at a lower temperature, $\mathrm{K}^{+}$ion distributions from the first step are negligibly affected. It was found that potassium-exchanged regions in glass substantially inhibit the silver ion exchange, so that patterned potassium waveguide regions can be used as a mask for the silver ion exchange. ${ }^{69,70}$ Combination of planar waveguide regions by exchanging these two ions into glass can be used to fabricate refractive planar waveguide lens elements ${ }^{71}$ or diffractive waveguide elements. ${ }^{72}$ Also, channel waveguides with different refractive index contrast values have been combined for fabrication of special devices, ${ }^{73}$ and channel waveguides fabricated using potassium/silver double ion exchange have shown decreased propagation losses. ${ }^{74}$

\section{Characterization of Ion-Exchanged Waveguide Properties}

Prism coupling ${ }^{75}$ is the most common characterization method for measuring propagation constants of planar waveguide modes. From the mode information, various approaches-generally variants of inverse WKB method - can be used to estimate the refractive index profile of the exchanged waveguide. ${ }^{76,77}$ The propagation constant values do not provide complete information about the waveguide profile; some assumptions about the nature of the profile need to be made, and then a best fit to measured values 
can be found. With different methods to retrieve the refractive index distribution, there is some disagreement about the accurate waveguide profile. ${ }^{78}$ Measurements can also be done for channel waveguides, though with more complication. ${ }^{50}$ Prism coupling can also be utilized to excite selected modes in the waveguide in order to measure individual mode propagation losses; the intensity distribution for each excited mode can then be observed at the waveguide end facet. The combined information on measured mode propagation constants and intensity distributions has been used to extract the refractive index profile. ${ }^{79}$

Waveguide profiles are often measured in deep planar waveguides. These are usually not representative of typical waveguides, as there is only limited interest in applications for planar waveguides. The actual target is to characterize diffusion profiles from ion exchange, so that more general waveguide processes can be modeled. Measured profiles can be utilized in parameter extraction-finding the parameters to model the ion exchange process-as detailed in Sec. 5.4. Even when extending such information from planar surface waveguides to complicated processes, such as two-step fabrication of single-mode buried channel waveguides may not achieve extremely accurate models. It can be sufficient to establish parameters for the fabrication of waveguide devices within required tolerances. More direct methods have also been used to measure waveguide profiles. These can be optical methods to measure directly the refractive index distribution, or material analysis methods to measure the ion concentration distributions from the ion exchange process. Refractive index profiles can be measured using a refractive near-field technique. ${ }^{80}$ Alternative optical measurement methods based, for example, on interferometry of thin slices of waveguide samples, ${ }^{55,81}$ have also been used. Also, the surface refractive index can be obtained from reflectivity measurements, and refractive index as a function of depth is then measured using a beveled surface ${ }^{29}$ or by exposing glass at different depths by etching. ${ }^{82}$

Several methods have been used to measure ion concentration profiles. One destructive method uses etching away of thin consecutive layers and obtaining ion concentrations from the etchant solution by means of spectroscopy ${ }^{83}$ or radiotracer analysis. Direct measurement of ion concentrations from the glass substrate edge facet can be obtained using an electron microprobe or scanning electron microscope for high-resolution scanning and analyzing the elements with energy dispersive $\mathrm{x}$-ray spectroscopy or backscattered electron analysis. $^{84}$

Waveguides are also typically characterized for loss and birefringence, and for coupling efficiency to optical fibers. Planar waveguide losses and birefringence can be obtained with prism coupling. For channel waveguides, propagation loss measurements are combined with coupling loss characterization, ${ }^{74}$ and birefringence can be measured as shown in Ref. 85.

\section{Modeling the lon Exchange Process}

The various fabrication processes described in Sec. 3 produce gradient-index profiles $n(x, y)$, where $x$ and $y$ are the transverse and depth directions, respectively. To properly describe the optical waveguiding properties of the ion-exchanged guides, it is first necessary to model the concentration profiles of the exchanging ions as a function of the processing conditions (temperature, exchange time, applied electric field, etc.). Phenomenologically, the diffusion of an ionic species within a glass network in response to a concentration gradient and an electric field is described by the Nernst-Planck drift-diffusion equation. However, in the case of binary, monovalent ion exchange, there are two additional factors to consider:

1. The constraint of local charge neutrality requires that the net ionic flux is zero (or equivalently that the total concentration of ionic species $A$ and $B$ is constant).

2. When the two interdiffusing ions have different diffusion coefficients-and therefore, by the NernstEinstein equation, different mobilities-any concentration gradient establishes an internal electric field, which exists even when the fabrication process is purely thermal and not field-assisted.

A rigorous derivation of the differential equation that governs binary ion diffusion is given in Ref. 86. Here, for the most part, we will derive it qualitatively. We label the in-diffusing and out-diffusing ionic species $A$ and $B$, respectively. For both species, the number flux of ions $\mathbf{j}_{i}(i=A, B)$ has components due to both diffusion in a concentration gradient and drift in an electric field. Applying the Nernst-Einstein equation for ionic conductivity, we have

$\mathbf{j}_{A}=-D_{A} \nabla c_{A}+\left(c_{A} D_{A} q / f k T\right) \mathbf{E}$,

$\mathbf{j}_{B}=-D_{B} \nabla c_{B}+\left(c_{B} D_{B} q / f k T\right) \mathbf{E}$.

Here, $c_{i}$ and $D_{i}$ are the concentration and diffusion coefficient of species $i$, respectively, $\mathbf{E}$ is the total electric field (the sum of the aforementioned internal field and any externally applied field), $T$ is the absolute temperature, $q$ is the proton charge, and $k$ is Boltzmann's constant. $f$ is a correlation factor (related to the Haven ratio) to account for the discrepancy in ion mobility values between the drift and diffusion phenomena. ${ }^{1,87}$ The local charge neutrality condition is met by requiring that $c_{A}+c_{B}=c_{0}$, the total concentration of mobile atoms in the glass. As $c_{0}$ is constant, we have a further constraint that

$\nabla c_{A}+\nabla c_{B}=0$.

Applying the continuity equation (Fick's Second Law),

$\frac{\partial c_{A}}{\partial t}+\nabla \cdot \vec{j}_{A}=0$

we are left with an expression for the time evolution of the in-diffusing ion concentration $c_{A}$. It is customary to use a normalized concentration $C=c_{A} / c_{0}$, which reads

$$
\begin{aligned}
\frac{\partial C}{\partial t}= & \frac{D}{1-(1-M) C}\left[\nabla^{2} C\right. \\
& \left.+\frac{(1-M)(\nabla C)^{2}}{1-(1-M) C}-\frac{q \mathbf{E}_{\mathrm{ext}}}{k T} \nabla C\right],
\end{aligned}
$$

where $M=D_{A} / D_{B}$, and we have relabelled $D_{A}$ as $D$. The three terms in square brackets indicate the contribution to concentration evolution due to diffusion, internal field drift, and external field $\left(\mathbf{E}_{\text {ext }}\right)$ drift, respectively. The use of a normalized concentration is justifiable because the index change is proportional to the concentration of the incoming ion, as will be shown shortly. 
In the above derivation, it is assumed that the self-diffusion coefficients $D_{A}$ and $D_{B}$-and therefore $M$ - are constant. In fact, these parameters are dependent on the local concentrations of each cation by the mixed-alkali effect ${ }^{88,89}$ (or, more accurately, the mixed mobile ion effect). ${ }^{90}$ Ionic motion is dependent on the probability of the ion possessing an activation energy $E_{a}$ required to jump between sites in the glass matrix. This relies on the local electrostatic and stress environment, which is in turn dependent on the local concentration of the exchanging ions. ${ }^{91,92}$ If the concentration dependences of $D_{i}$ are known and if they vary slowly over the area of the waveguide, numerical solutions of Eq. (4) are still valid if the values of $D_{i}$ are updated at each time step. Otherwise, more rigorous formulations $\mathrm{s}^{90,93}$ of Eq. (4) can be used, which explicitly account for gradients in $D_{i}$ in the divergence of ionic flux.

\subsection{Solutions of the Diffusion Equation}

Equation (4) holds true in any number of spatial dimensions. While 1D (slab) and 2D (channel) waveguide modeling are very common, 3D modeling is rarely needed-as ion-exchanged waveguides are weakly guiding, the waveguide geometry changes very slowly in the propagation direction and therefore both drift and diffusion in this direction can be considered negligible. However, full 3D modeling is necessary when ion exchange is used to create diffractive elements. ${ }^{94,95}$ For slab waveguides, various approximate analytical solutions to Eq. (4) have been suggested under limiting cases of $M$ and the applied field. With $M=1$ and no applied field - and neglecting any concentration dependence of ion mobility - the binary diffusion equation reduces to the simple form

$\frac{\partial C}{\partial t}=D \frac{\partial^{2} C}{\partial y^{2}}$,

which has the solution $C(y, t)=C_{0} \operatorname{erfc}(y / 2 \sqrt{D t})$, where erfc is the complementary error function and $C_{0}$ is a constant. For a field-assisted process in which drift dominates over diffusion, a modified Fermi distribution has been assumed for the ion concentration. ${ }^{96}$ Otherwise, for arbitrary processing parameters, Eq. (4) can be easily solved in 1D using a variety of numerical methods. Here, we concentrate on the more substantial problem of channel waveguide modeling, which imposes stringent requirements on the stability and speed of the computational algorithm. Generally speaking, small values of $M$ lead to computational instability near the glass/melt interface, where two terms in the denominator of Eq. (4) become very small. Early efforts at channel waveguide modeling used the explicit Dufort-Frankel algorithm. ${ }^{86}$ However, this method is stable only for values of $M$ greater than about 0.1 , which excludes several practical ion exchange systems including that of $\mathrm{Ag}^{+}-\mathrm{Na}^{+}$exchange in many silicate glasses. It has been shown ${ }^{97,98}$ that the alternating direction implicit method of Peaceman and Rachford ${ }^{99}$ is stable down to very low values of $M$. The speed improvement over fully implicit methods such as Crank-Nicolson permits the use of a very fine computational mesh in both space and time. Improvements using adaptive meshes have been presented. ${ }^{100}$

Boundary conditions on all four edges of the 2D computational domain must be imposed. For all processing steps, at the bottom of the domain (not necessarily the back side of the substrate, but a distance far enough from the surface such that the concentration of in-diffusing atoms is negligible), we force $C=0$. An identical condition is found on the two sides of the domain; these must be sufficiently far from the mask opening that transverse diffusion does not impact these boundaries. The boundaries must be a few $\sqrt{D t}$ from the edge of the opening, for example. On the top surface of the substrate, the boundary condition on $C$ depends on the particular fabrication process. In the case of thermal exchange from a molten salt, the mask serves to block any diffusion normal to the surface, leading to the Neumann condition $(\partial C / \partial y$ $=0$ ), while the open regions of the mask permit a known normalized concentration $C=1$. For field-assisted burial, as the top surface is exposed to salts that lack ion $A$, we have $C$ $=0$ everywhere on this surface. In the annealing procedure, we again have $\partial C / \partial y=0$ on the top surface, as no ions of either type may enter or exit from the surface of the substrate. The $C=1$ boundary condition on the open regions of the mask is justified because the time required for the ionic concentrations to reach equilibrium at the surface is very short in comparison to the time scales used in waveguide fabrication. ${ }^{101}$ Although it is possible to determine the actual surface concentration as a function of the glass and melt composition by thermodynamic means, ${ }^{102}$ for practical reasons it is simpler to use the normalized value and correlate it to an empirically determined surface index change, as we will discuss in Secs. 5.3 and 5.4.

\subsection{Modeling the Electric Field}

When an externally applied electric field is present, its spatial profile $\mathbf{E}_{\text {ext }}(x, y)$ is critically related to the evolution of ion concentration. To a first approximation, the field can be considered constant in time and expressed as the gradient of a potential profile $\varphi(x, y)$ that is calculated by Laplace's equation. However, when $M \neq 1$, the existence of a spatial variation in ion concentration implies a proportional variation in electrical conductivity $\sigma(x, y)$, via the Nernst-Einstein relation. In this case, $\varphi(x, y)$ satisfies the nonstandard Laplace equation,

$\sigma(x, y) \nabla^{2} \varphi(x, y)+\nabla \sigma(x, y) \cdot \nabla \varphi(x, y)=0$.

If we assume a linear relationship between ion concentration and conductivity, then

$\sigma(x, y) \propto \frac{1}{M}[1-C(x, y)]+C(x, y)$,

where the proportionality constant is irrelevant due to the mathematical homogeneity of Eq. (6). As $C(x, y)$ evolves over time, so does $\sigma(x, y)$ and thus also $\varphi(x, y)$ and $\mathbf{E}_{\mathrm{ext}}(x, y)$ -although over lengthy time scales, such that electrostatic modeling remains appropriate. Therefore, it is necessary to update the field profile at each time step. The numerical solution of Eq. (6) in a form well-suited to applying to Eq. (4) can be found in Ref. 53. The boundary conditions on $\varphi$ are set such that $\varphi=0$ at the bottom of the domain and $\partial \varphi / \partial x=0$ at the sides of the domain (we extend the domain far enough from the edges of any mask that the field lines can be assumed parallel to the sides of the domain). On the top side, in all regions where the mask is open, $\varphi=V h / d$, where $V$ is the voltage applied across the substrate, and $h, d$ are the thickness of the domain and the thickness of the substrate, respectively. In regions where the mask is closed, we have 
the Neumann condition $\partial \varphi / \partial y=0$, since the mask serves as a barrier to ion motion normal to the surface.

Qualitatively, when $M<1$, the concentration dependence of $\sigma$ perturbs the electric field lines such that they tend to be excluded from the center of the diffused waveguide. This has been shown to manifest as a noticeable difference in the depth of waveguides buried in a field-assisted process, depending on their pre-burial width. ${ }^{54}$

\subsection{Converting Ion Concentration to Refractive Index Profile}

The final step in modeling ion-exchanged waveguides is to convert the exchanged ion distribution $C(x, y)$ to a refractive index profile $n(x, y, \lambda)$. The exchanged ions locally affect the refractive index by three physical mechanisms; ionic size, ionic polarizability, and photoelastic effects due to induced stress. ${ }^{103}$ While the latter mechanism has been found to dominate the index change for $\mathrm{K}^{+}-\mathrm{Na}^{+}$ion-exchanged guides, it is small in comparison to the other two effects in $\mathrm{Ag}^{+}-\mathrm{Na}^{+}$guides. ${ }^{59,60}$ For small absolute concentrations of the exchanging ion, it is relatively easy to show that there is a linear relationship between exchanged ion concentration and index change. ${ }^{104}$ This follows from the Lorentz-Lorenz equation, which relates the refractive index $n$ of a multicomponent dielectric to its polarizability

$\frac{n^{2}-1}{n^{2}+2}=\frac{4 \pi}{3} \sum_{i} N_{i} \alpha_{i}$,

where $N_{i}$ and $\alpha_{i}$ are the number density and polarization volume of component $i$, respectively. The components are incoming species $A$, outgoing species $B$, and all other components, indexed by $j$. If we assume that the exchange process substitutes a number density $\Delta N$ of species $B$ with an identical number density of species $A$, then the Lorentz-Lorenz equation for index $n$ (after the exchange) and $n_{\text {sub }}$ (before the exchange) are, respectively,

$$
\begin{aligned}
\frac{n^{2}-1}{n^{2}+2}= & \frac{4 \pi}{3}\left[\left(N_{A}+\Delta N\right) \alpha_{A}+\left(N_{B}-\Delta N\right) \alpha_{B}\right. \\
& \left.+\sum_{j} N_{j} \alpha_{j}\right]
\end{aligned}
$$

and

$\frac{n_{\mathrm{sub}}^{2}-1}{n_{\mathrm{sub}}^{2}+2}=\frac{4 \pi}{3}\left[N_{A} \alpha_{A}+N_{B} \alpha_{B}+\sum_{j} N_{j} \alpha_{j}\right]$.

Subtracting these two equations and assuming that $\Delta n=n$ $-n_{\text {sub }} \ll 1$ produces the relationship

$\Delta n=\frac{2 \pi\left(n_{\mathrm{sub}}^{2}+2\right)}{3 n_{\mathrm{sub}}}\left(\alpha_{A}-\alpha_{B}\right) \Delta N$,

which shows that $\Delta n$ is linearly proportional to $\Delta N$ (and that it can in fact be negative if $\alpha_{A}<\alpha_{B}$ ).

In practice, one does not to need to know the ionic polarizability volumes $\alpha_{A}$ and $\alpha_{B}$ or the absolute value of $\Delta N$. Instead, an empirical value of the maximum index change $\Delta n_{0}$ is determined for a given glass/salt melt combination, and this is correlated to the normalized concentration $C=1$. Bearing in mind that this index change is wavelengthdependent, the index profile can therefore be expressed as

$n(x, y, \lambda)=n_{\mathrm{sub}}(\lambda)+\Delta n(x, y)=n_{\mathrm{sub}}(\lambda)+\Delta n_{0}(\lambda) C(x, y)$.

\subsection{Empirical Determination of the lon Exchange Parameters $D, M$, and $\Delta n_{0}$}

Sections 5.1 to 5.3 suggest that modeling of the ion exchange process requires accurate values of $D, M$, and $\Delta n_{0}$. These parameters are not routinely provided by glass manufacturersand in any case the first two are strongly dependent on the processing temperature while the third depends considerably on the composition of the salt melt. Furthermore, even different batches of the same glass substrates may show a wide variation in the diffusion coefficients, depending on the annealing history of the glass; this can be evident when characterizing substrates of different diameter, for example.

Determination of ion exchange parameters can be achieved experimentally by examining the characteristics of slab waveguides under various exchange temperatures and times, where the effective indices of the slab modes are measured using prism coupling (M-line) techniques. ${ }^{75}$ This is not a trivial task, as even the one-dimensional profile $C(y)$ has an unknown form when there is no knowledge of $M$ - this prevents direct calculation of the parameters by least-squares fitting to the measured index profile. In the most common approach, ${ }^{105,106} \mathrm{Eq}$. (4) is solved in 1D using assumed values of $D$ and $M$, and the resulting concentration profile is converted to an index profile using an assumed value of $\Delta n_{0}$. The effective indices of this modeled slab waveguide are calculated using WKB methods, ${ }^{107}$ and compared to the measured effective indices. This process is iterated, adjusting the three parameters to minimize the squared error between measured and modeled indices; using a highly multimode waveguide for this procedure is suggested so as to more accurately resolve the index profile. More recently, the optimization has been automated using a genetic algorithm. ${ }^{108}$ A more direct alternative to the M-line technique is to measure the concentration profile by scanning electron microscopy, or the index profile by near-field refractometry. ${ }^{84}$

Over a wide range of processing temperatures, the selfdiffusion coefficients show an Arrhenius temperature dependence,

$D(T)=D_{0} e^{-E_{a} / k T}$,

where $E_{a}$ is the energy barrier required for species $A$ to jump between sites and $D_{0}$ is a constant. When calculating $D_{0}$ and $E_{a}$ by the afore-mentioned procedure, it is beneficial to characterize slab waveguides processed over a range of temperatures. A linear fit of $\ln (D)$ versus $1 / T$ allows one to extrapolate $D(T)$ to temperatures for which fabricating a highly multimode waveguide by thermal ion exchange would be impractical; for instance, temperatures used for the thermal annealing process. In addition, concentration dependence of the self-diffusion coefficients can be calculated with high accuracy using Boltzmann-Matano analysis. ${ }^{84}$

\section{Guided-Wave Devices by lon Exchange}

\subsection{Passive Devices}

The early demonstrations of ion-exchanged waveguide devices included multimode devices such as splitters and 
wavelength multiplexers. Broader functionalities are provided by single-mode devices; these are also more applicable to contemporary applications that use single-mode fibers. First demonstrators of splitters, couplers, tapers, polarizers, ring resonators, and thermo-optic switches were discussed in Sec. 2. Virtually any passive waveguide device structure can be fabricated by ion exchange, including Mach-Zehnder interferometers, ${ }^{109}$ multimode interference (MMI) couplers $^{110,111}$ and phased-array multiplexers. ${ }^{112}$ 1-to-N (or 2-to-N) splitters based on cascaded single-mode waveguide Y-branches provide advantages of low imbalance, broadband operation, and polarization insensitivity due to inherently symmetric splitting. Directional couplers and Mach-Zehnder interferometers as wavelength splitters/combiners have been compared. ${ }^{113}$ MMI couplers are usually based on high index-contrast step waveguides, but they have been demonstrated by ion exchange, although special considerations need to be taken into account in their design. ${ }^{114,115}$ In arrayed-waveguide grating (AWG) multiplexers, ion-exchanged waveguides provide low polarization sensitivity ${ }^{112}$ and thermal fine-tuning of the device response is possible. ${ }^{116}$ The thermo-optic effect can be utilized in variable attenuator devices; an ion-exchanged Mach-Zehnder structure variable attenuator was demonstrated with $1 \mathrm{~dB}$ insertion loss, dynamic range of $38 \mathrm{~dB}$, and polarization dependent loss varying from 0.2 to $0.6 \mathrm{~dB}$ in the attenuation range of 10 to $20 \mathrm{~dB} .^{117}$ The maximum power consumption of the device was $138 \mathrm{~mW}$.

To form optical gratings within ion-exchanged waveguides, a photowriting technique similar to fiber Bragg grating fabrication can be used. ${ }^{118,119}$ Various ion-exchanged glasses have been found to have sufficient photosensitivity at UV wavelengths, including the BGG31 glass developed for ion exchange. ${ }^{120}$ An add-drop multiplexer has been demonstrated utilizing a Bragg grating in a two-mode waveguide between asymmetric Y-branches; the grating couples light between even and odd modes of the waveguide. ${ }^{121}$ An adiabatic broadband wavelength multiplexer was realized by patterning a periodically segmented waveguide into a Y-branch configuration. ${ }^{122}$

The ultimate target of integrated optics is integration of multiple guided-wave devices and circuits onto a single substrate. For example, 1-to-N splitters and AWGs already combine multiple basic waveguide elements, and integration of Mach-Zehnder filter devices with 1-to-8 splitting was demonstrated in Ref. 123. An interesting possibility is to utilize waveguide burial processes in order to integrate multiple waveguide circuit layers into a single substrate. This was demonstrated by combining silver-sodium exchanged surface waveguides with buried waveguides at the depth of $22 \mu \mathrm{m}^{124}$

Early examples of hybrid integration with semiconductor devices are photodetectors integrated on glass substrates with ion-exchanged waveguides. In 1987, MacDonald et al. demonstrated a hybrid optoelectronic circuit, in which GaAs photodetectors were bonded to a metallic interconnect layer deposited and patterned on a glass substrate with channel waveguides exchanged from Ag thin film sources. ${ }^{125}$ Larson and Phelan produced, hydrogenated amorphous Si photodetectors deposited on potassium-exchanged glass waveguides, ${ }^{126}$ followed by similar detectors based on germanium to reach longer wavelengths. ${ }^{127}$ Yi-Yan et al. introduced a liftoff technique, by which thin III-V semi- conductor multilayer membranes could be bonded on top of a glass substrate with ion-exchanged waveguides, and metal-semiconductor-metal detectors were fabricated in this structure. $^{128}$

\subsection{Nonlinear Waveguide Devices}

There was substantial growth of interest in optical nonlinearities in waveguides in the mid-1980s, and by then ion exchange had been established as a reliable method for glass waveguide fabrication. Semiconductor-doped glasses were a promising material, and potassium-sodium exchange was used to make low-loss waveguides for nonlinearity studies. ${ }^{129}$ As commercially available filter glasses had low sodium concentration, a sodium-rich melt was used in this study -alternatively, waveguides could be fabricated in commercial glasses by cesium-potassium exchange. Degenerate fourwave mixing from fast nonlinearity in CdSSe-doped glass was observed by prism-coupling pump and probe beams into a planar waveguide. ${ }^{130} \mathrm{An}$ ion-exchanged directional coupler in such a glass was demonstrated with picosecond all-optical switching, but this was dominated by fast saturable absorption, so the switching fraction was not high. ${ }^{131}$ The limitation was that these commercial glass types did not have optimal nonlinear properties, as the semiconductor nanocrystals in the glass did not have sufficient size distributions. More recently, silver-sodium and potassium-sodium ion-exchange waveguide fabrication was demonstrated in glasses doped with $\mathrm{PbS}$ quantum dots, which have high nonlinearities. ${ }^{132}$ The quantum-dot optical properties remained intact after ion exchange. However, practical nonlinear devices would need low waveguide propagation losses, requiring better optical quality of glass than was available in the sample amount produced.

Aitchison et al. demonstrated spatial optical solitons ${ }^{133}$ and soliton interaction ${ }^{134}$ in potassium-sodium ionexchanged planar waveguide fabricated on B270-glass, which does not have a particularly high nonlinearity, but it has low losses. They also made similar observations in thallium-potassium-exchanged experimental glass containing niobium and titanium, having higher nonlinearity. ${ }^{135}$

\subsection{Lasers and Amplifiers}

In recent years the research on active rare-earth-doped waveguide lasers and amplifiers has been the most dynamic area of ion-exchanged glass waveguides. Amplification in ion-exchanged waveguides was demonstrated as early as 1973 by Saruwatari et al. They demonstrated a waveguide laser in a borosilicate glass containing $4.0 \mathrm{wt} . \% \mathrm{Nd}_{2} \mathrm{O}_{3}$. The waveguide used was highly multimode and was fabricated with a field-assisted burial process. ${ }^{23}$ Not much happened in the field for more than a decade until, largely inspired by the promise of rare-earth-doped fibers, the field was "re-born" in $1989 .{ }^{136}$ In the early 1990s several studies were reported, demonstrating mostly Nd-doped waveguide lasers and amplifiers operating at $\sim 1.06 \mu \mathrm{m}$ wavelength. ${ }^{137-142}$ However, with the emerging applications of Er-doped fiber amplifiers for the wavelength division multiplexing (WDM) networks operating at $1.55 \mu \mathrm{m}$, the focus on planar rare-earth-doped waveguides also turned into Er-doped devices. With ionexchanged waveguides the most success has been achieved using phosphate glasses as a substrate, as they can be doped with large amounts of rare earth ions. Silicate glasses suffer 
from the relatively poor solubility of rare-earth-ions, which leads to clustering and a reduction in conversion efficiency. ${ }^{143}$ The use of highly-doped phosphate glasses enables the fabrication of compact devices, i.e., amplifiers with lengths of only a few centimeters and short-cavity waveguide lasers suitable for single-frequency operation.

In the development of Er-doped waveguide amplifiers the major breakthrough occurred in 1995, when Barbier et al. demonstrated an amplifier producing $7 \mathrm{~dB}$ of net gain in a double-pass configuration, with a device length of only $41 \mathrm{~mm}$ in an Er/Yb-codoped phosphate glass substrate. ${ }^{144}$ They used a field-assisted burial process to reduce the fiberto-waveguide coupling loss; this also reduces the waveguide propagation losses as the interaction with the glass surface is avoided. With phosphate glasses this is of high importance, since phosphate glasses are known to exhibit poor chemical durability when exposed to the molten salts used in conventional ion exchange. Nevertheless, using special phosphate glasses with improved chemical durability, high quality surface waveguides have also been demonstrated. ${ }^{145,146}$ Alternatively, the dry silver ion exchange process can be used in fabricating high-quality surface waveguides, ${ }^{147}$ and indeed, Patel et al. employed the Ag-film ion exchange process in achieving the record high gain per unit length. ${ }^{148}$ They demonstrated a peak gain of $4.1 \mathrm{~dB}$ at $1534-\mathrm{nm}$ wavelength in a 3-mm long phosphate glass waveguide, which was doped highly with $\mathrm{Er}(8 \mathrm{wt} . \%)$ and $\mathrm{Yb}(12 \mathrm{wt}$. \%). Following their initial demonstration, Barbier et al. proved the feasibility of Er-doped waveguide amplifiers (EDWAs) by reporting several impressive demonstrations of EDWAs. The demonstrations include an amplifying 4-wavelength combiner ${ }^{149}$ and a successful operation of EDWAs in high-speed multiwavelength system experiments. ${ }^{150}$ The results of Barbier et al. led to the commercialization of a family of EDWA-products by Teem Photonics. ${ }^{151}$

After initial demonstrations of waveguide lasers operating at $1.06 \mu \mathrm{m}$ wavelength, the research started to aim at Er-doped waveguide lasers. There was a need in WDM technology for cheap and compact transmitters in the 1550 $\mathrm{nm}$ wavelength region. The first ion-exchanged waveguide laser emitting at $1540 \mathrm{~nm}$ wavelength was demonstrated by Feuchter et al. ${ }^{152}$ The substrate used was a silicate glass BK7 with 0.5 wt. $\% \mathrm{Er}_{2} \mathrm{O}_{3}$, and a thermal ion exchange in a $\mathrm{KNO}_{3}$ bath was employed in waveguide fabrication. The laser cavity was formed by bonding dielectric mirrors to the chip end facets. However, as with amplifiers it was clear that the use of phosphate glasses would enable much improved performance. Also, it was important to integrate waveguide gratings with the cavity in order to achieve single frequency operation. Indeed, several multi-wavelength waveguide lasers arrays using $\mathrm{Er} / \mathrm{Yb}$-codoped phosphate glass substrates and surface relief-type gratings have been demonstrated. ${ }^{145,146,153}$ Veasey et al. ${ }^{145}$ used potassium ion exchange and a distributed Bragg reflector (DBR) grating, Madasamy et al. ${ }^{153}$ employed Ag-film ion-exchanged waveguides and a DBR configuration, and Blaize et al. ${ }^{146}$ used molten salt silver ion exchange with a DFB-grating configuration. In these approaches, a single grating was employed and the multiwavelength operation was achieved by changing the waveguide width within the array, resulting in different effective indices for the waveguide channels. To illustrate the potential of Erdoped waveguide lasers, Veasey et al. achieved an output power of $80 \mathrm{~mW}$ at $\sim 1536-\mathrm{nm}$ wavelength with $350 \mathrm{~mW}$ of coupled pump power at 977-nm wavelength. Furthermore, the laser linewidth of only $3 \mathrm{kHz}$ has been demonstrated by Bastard et al. ${ }^{154}$

Most waveguide laser demonstrations in phosphate glass utilize Bragg gratings fabricated by lithographic procedures or thin-film reflectors attached directly to the waveguide facets. Both of these cavity mirror solutions involve multiple steps in the clean room, and are therefore time-consuming and expensive approaches. This can be avoided by utilization of Bragg grating inscription into phosphate glass by UV irradiation through a phase mask. UV-written Bragg gratings are nowadays widely deployed in fibers and fiber lasers but the use of UV-written Bragg gratings in ion-exchanged waveguide lasers was delayed. It had turned out to be difficult to write gratings in a glass waveguide material that provides enough gain in short cavity lengths (i.e., a phosphate glass waveguide). Note that the UV-written Bragg gratings are also important in improving the laser efficiency since UVwritten volume Bragg gratings are nearly lossless and do not couple pump laser light out from surface waveguides. The first experiments on photosensitivity of ion-exchanged Er/Yb-codoped phosphate glass were reported by Pissadakis et al. ${ }^{155}$ They demonstrated a thin surface grating operating in the Raman-Nath region with a refractive index change of $2 \times 10^{-3}$. Only a small index change on the order of $10^{-5}$ was observed in a pristine sample. This kind of a surface grating would not work as a mirror in waveguide lasers for two reasons: 1. a surface grating at the pump input side would couple out the pump laser power at $\sim 980 \mathrm{~nm}$ propagating close to the surface; 2 . a surface grating with a relatively low index modulation would not provide enough optical feedback for lasing. In 2006 Yliniemi et al. reported high quality UV-written Bragg gratings in ion-exchanged phosphate glass and demonstrated a waveguide laser utilizing such a grating. ${ }^{156,157}$ In these studies commercially available Schott IOG-1 phosphate glass was used.

The studies of Yliniemi et al. ${ }^{156,157}$ revealed that fabrication of a volume grating in Er/Yb-codoped phosphate glass, suitable for high quality waveguide fabrication, is indeed a complex task. Volume waveguide gratings with maximum reflectance of only about $15 \%$ were obtained. ${ }^{157}$ For typical laser applications, however, reflectance above $70 \%$ should be obtained, which is why another approach was adopted. It was demonstrated that narrow-band high reflectance waveguide gratings can be written in an undoped IOG-1 glass; therefore, a hybrid IOG-1 glass was utilized. The hybrid glass is a monolithic substrate composed of an Er/Yb-codoped part and an undoped part, bonded to each other. ${ }^{158}$ Yliniemi et al. formed the Bragg grating in the undoped part of the hybrid substrate, and it served as the wavelength selective cavity mirror as well as the output coupler of the laser. The $\mathrm{Er} / \mathrm{Yb}$-codoped part of the hybrid glass provided the gain required for the laser operation. The Bragg grating was written through a phase mask with an ArF pulsed excimer laser (wavelength of $193 \mathrm{~nm}$ ). A phase mask with a period of 1065 $\mathrm{nm}$ was designed so that the Bragg grating in IOG-1 glass would have a peak reflectance at $\sim 1535 \mathrm{~nm}$, corresponding to the gain maximum of the Er-doped glass. Furthermore, a key finding was that the grating had to be written prior to the fabrication of the waveguide. This way, the large absorption at the writing wavelength induced by the waveguide is avoided and the grating planes extend deep below the surface of the glass substrate. 
In laser experiments of Ref. 157, a fiber coated with an $\mathrm{SiO}_{2} / \mathrm{TiO}_{2}$-thin film stack aligned with the waveguide facet in the Er-Yb-codoped part of the substrate was used as the other cavity mirror. Pump power from a fiber-pigtailed semiconductor diode laser emitting at $980 \mathrm{~nm}$ was also delivered through this fiber. The thin film stack was designed to have transmittance close to $100 \%$ at the pump wavelength of 980 $\mathrm{nm}$ and reflectance close to $100 \%$ at 1550 -nm wavelength region. The demonstrated single-frequency laser operated with an output power of $9.0 \mathrm{~mW}$ with a pump power of $200 \mathrm{~mW}$ and a $135 \mathrm{~mW}$ threshold power. The slope efficiency was $13.9 \%$.

\section{Additional Applications of lon-Exchanged Waveguide Devices and Circuits}

Fiber-optic passive components based on single-mode ionexchanged glass waveguides are the most obvious applications of this technology. In particular, 1-to-N and 2-to-N splitters/combiners have been available for many years from different vendors. A primary application has been in branching devices for passive optical networks and for multiplexing signals at different wavelengths prior to optical amplification in WDM transmission systems. The performance of these devices is excellent, so their prospects depend largely on emerging market needs, and competitiveness of alternate solutions, based on other waveguide technologies, or microoptical or fiber components. Other passive guided-wave devices and circuits-for example those targeting WDM applications ${ }^{116}$ — could be based on similar fabrication processes. A key question here is whether such components can achieve sufficient performance and reliability at competitive fabrication cost.

The next step for penetration of optical communication into shorter distances is the replacement of copper interconnects in backplanes and circuit boards, where with increasing data rates, optics could provide a high bandwidthdistance product with low signal interference and power consumption. Multimode optical waveguides represent a lowcost interconnect medium. While this work has mostly concentrated on polymer waveguide technologies, more recently ion-exchanged waveguides in glass sheets laminated onto circuit boards have been suggested. ${ }^{159}$ Thin, high-quality glass sheets in large sizes have increasingly become available for display applications, and glass is in many respects an ideal board material with excellent thermal, high frequency dielectric, mechanical, environmental, and optical properties. ${ }^{160,161}$ High density of glass waveguides can be realized by fabricating them on both sides of a glass sheet. ${ }^{162}$ As is well known with optical multimode fibers, graded-index waveguides can support much higher bandwidth-distance products than step-index waveguides due to reduced intermodal dispersion, which would make ion-exchanged waveguide interconnects quite future-proof, which can be important, as bandwidth requirements are constantly increasing.

After optical communication, the most interesting application area for guided-wave devices is in optical sensors. It needs to be remembered that a significant portion of the sensor market exists at price levels significantly lower than even low-cost waveguide technologies can reach, so that there is a large gap from demonstration of sensor concepts to commercial products. Several sensor concepts have been demonstrated using ion-exchanged waveguides, ${ }^{163}$ many using guided-wave interferometer structures, which can be used for example in distance or refractive index measurements. There are also sensor concepts utilizing multimode waveguides, ${ }^{164}$ which can provide cost advantages. One main category is chemical sensors, ${ }^{165}$ probing the sample substance with light propagating in waveguides. These are often based on evanescent-field sensing, measuring a thin layer at the waveguide surface. Ion-exchanged waveguides have relatively low refractive index differences, particularly in comparison with refractive index difference between glass substrate and the measurand-typically a liquid substance, with a much lower refractive index value than glass. For this reason, they do not have very high sensitivity for evanescentfield sensing, as the mode extends deeper into the substrate than it does above the surface. Evanescent field enhancement can be achieved using a thin high-index film on top of the waveguide, ${ }^{166}$ effectively creating a dielectric-loaded waveguide. Alternatively, metal films have been incorporated to couple light into surface plasmons that have a high portion of evanescent field. ${ }^{167}$ Development of integrated optics sensors basically needs the support of waveguide device foundries, with sensor platforms available that can be modified for various sensing applications. One example of a platform for lab-on-chip microdevices has a glass substrate integrating ion-exchanged waveguides for optical sensing and etched microfluidic channels, with polydimethylsiloxane cover. ${ }^{168,169}$

A novel application has recently been found in astronomy, particularly in astronomical interference, for beam combining in visible or infrared observations. A series of papers studied this approach using ion-exchanged waveguide devices as an enabling technology: first, proposing the approach and developing the concept ${ }^{170}$ feasibility was demonstrated in laboratory with off-the-shelf components ${ }^{171}$ and characterization of a beam combiner validated the technology, ${ }^{172}$ followed by astronomical interference observations ${ }^{173}$ at $1.6-\mu \mathrm{m}$ wavelength and then by development of new waveguide devices for longer wavelength $(2.0$ to $2.5 \mu \mathrm{m})$ observations. ${ }^{174}$ In astronomy, there is high interest in going further to midinfrared spectrum, ${ }^{175}$ and ion-exchanged waveguides have been demonstrated in germanate glasses targeting the 3 to 4- $\mu \mathrm{m}$ spectral range. ${ }^{176,177}$

Here, ion exchange has been a potential fabrication technology for final realization of such applications, but also to some extent an available platform for carrying out scientific experiments. Some further examples of using ionexchanged waveguides as a platform for demonstrations and studies can be given. Optical propulsion of polystyrene microspheres suspended in fluid by evanescent field of singlemode waveguides made by $\mathrm{Cs}^{+}$ion-exchange in glass were studied. ${ }^{178}$ Another example is measurement of the standing wave pattern of counterpropagating waveguide modes in millimeter scale by an optical near-field microscope. ${ }^{179}$ Here, the focus was not the characterization of particular silver ionexchanged waveguides, but general demonstration of instrumentation. Ion-exchanged waveguides have also been used for evanescent field fluorescence microscopy, utilizing different penetration depth for evanescent field from various modes excited in multimode silver-exchanged waveguides. ${ }^{180}$

\section{Outlook and Conclusion}

Ion-exchanged waveguide components are available for single-mode fiber communication applications, primarily as splitters with high splitting ratios. Their eventual success 
will depend on the expansion of markets, and on whether they provide cost or performance advantages over the main competitors, which are expected to be components fabricated using other passive integrated optics technologies.

Ion-exchanged waveguide amplifiers have been extensively investigated and very impressive results have been demonstrated. However, so far they have achieved little practical success. Two major factors contribute to this: 1. the short length of waveguide devices requires a very high gain per unit length in order to compete with fiber amplifiers. This necessitates a high concentration of rare earth ions within the glass, which eventually leads to a decrease in pumping efficiency due to cooperative up-conversion; 2 . unlike rare earth-doped fibers, which have radial concentration and index profiles that are optimized for pump/signal/gain medium overlap, waveguides that are ion-exchanged in a homogeneously doped substrate exhibit a relatively poor overlap, decreasing the gain. However, waveguide amplifiers do have an advantage over their fiber-based counterparts in that the passive elements (pump-signal combiner, tap coupler for power monitoring, etc.) can be monolithically integrated onto the same substrate with the amplifier, which is enabled by the hybrid glass. ${ }^{158}$ It remains to be seen whether these compact and potentially very low-cost amplifiers will find applications in telecommunications. Ion-exchanged waveguide lasers, however, are becoming increasingly popular. They have potential use in a wide variety of applications, requiring, e.g., a very narrow linewidth. Here also, the use of the hybrid glass can bring additional advantages in terms of increased functionality and cost due to monolithic integration (see, e.g., Madasamy et al. ${ }^{181}$ ). Another example of integrating amplification into device functionality is ring resonator with gain, fabricated into Nd-doped glass by silver ion exchange, which can be used as an optical gyroscope rotation rate sensor. ${ }^{182}$ The finesse of a $16 \mathrm{~mm}$ diameter resonator increased from 11 to 250 , with pumping of the gain medium.

For the other application areas mentioned, the question is whether ion-exchanged waveguide technology can meet the final requirements for each application, and if required product volumes will be high enough for volume technologies of integrated optics. The required volumes may depend on whether common technology platforms supported by foundries will be available for multiple products.

Ion-exchanged waveguides will certainly continue to be used in research work-in fact, several of the research efforts described here are still ongoing. One particular direction expected to be of future interest is hybrid/heterogeneous integration of glass waveguides with various materials to optimally reach more diverse functionality. For example, nonlinear polymer cover materials have been deposited on top of ion-exchanged waveguides to make nonlinear hybrid waveguide structures in which second harmonic generation ${ }^{183}$ and parametric amplification ${ }^{184}$ have been demonstrated. Also, with a photochromic polymer on an ion-exchanged MachZehnder interferometer, it was shown that the phase of guided wave could be modulated with an optical control beam. ${ }^{185}$ A more recent example is a modulator combining Ag-film exchanged waveguides with an electro-optic polymer layer, using a fabrication process that aligned the hybrid waveguide with coplanar modulation electrodes using a single photolithographic masking step. ${ }^{186}$

To conclude, the authors expect that, due to its simplicity, ion-exchanged waveguide technology will continue to serve as an important platform in testing new ideas and demonstrating new device concepts. As for commercial applications, ion-exchanged splitting devices for fiber optic communications may have an important role as the markets expand. It is also believed that the technology will remain an important choice for several "niche" applications, including waveguide lasers and a wide variety of sensors. Here, the hybrid approaches discussed above are particularly promising.

\section{References}

1. R. Terai and R. Hayami, "Ionic diffusion in glasses," J. Non-Cryst. Solids 18(2), 217-264 (1975).

2. R. H. Doremus, "Ion exchange in glass," Chapter 1 in Ion ExchangeA Series of Advances, Vol. 2, J. A. Marinsky, Ed., pp. 1-42, Marcel Dekker, New York (1969)

3. T. Findakly, "Glass waveguides by ion exchange: a review," Opt. Eng. 24(2), 244-250 (1985)

4. R. V. Ramaswamy and R. Srivastava, "Ion-exchanged glass waveguides: a review," IEEE J. Lightwave Technol. LT-6(6), 984-1000 (1988).

5. L. Ross, "Integrated optical components in substrate glasses," Glastech. Ber. 62(8), 285-297 (1989).

6. S. I. Najafi, Introduction to Glass Integrated Optics, Artech House, Boston, MA (1992).

7. N. V. Nikonorov and G. T. Petrovskii, "Ion-exchanged glasses in integrated optics: the current state of research and prospects (a review)," Glass Phys. Chem. 25(1), 16-55 (1999).

8. B. West, "Ion-exchanged glass waveguides," in The Handbook of Photonics, 2nd ed., M. C. Gupta and J. Ballato, Eds., pp. 13-1-13-35, CRC Press, Boca Raton (2006).

9. J.-E. Broquin, "Glass integrated optics: state of the art and position toward other technologies," Proc. SPIE 6475, 647507 (2007).

10. P. Mazzoldi and C. Sada, "A trip in the history and evolution of ionexchange process," Mat. Sci. Eng. B 149(2), 112-117 (2008).

11. A. Puche-Roig, V. P. Martin, S. Murcia-Mascaros, and R. I. Puchades, "Float glass colouring by ion exchange," in Supplement $1,2 n d$ International Congress on Glass Science in Art and Conservation, J. Cultural Heritage 9, e129-e133 (2008).

12. A. Y. Zhang, T. Suetsugu, and K. Kadono, "Incorporation of silver into soda-lime silicate glass by a classical staining process," J. Non-Cryst. Solids 353(1), 44-50 (2007).

13. T. Pradell, J. Molera, J. Roque, M. Vendrell-Saz, A. D. Smith, E. Pantos, and D. Crespo, "Ionic-exchange mechanism in the formation of medieval luster decorations," J. Am. Ceram. Soc. 88(5), 1281-1289 (2005).

14. G. Schulze, "Versuche über die diffusion von silber in glas," Ann. Phys. 345(2), 335-367 (1913).

15. S. S. Kistler, "Stresses in glass produced by nonuniform exchange of monovalent Ions," J. Am. Ceram. Soc. 45(2), 59-68 (1962).

16. A. K. Varshneya, "Chemical strengthening of glass: lessons learned and yet to be learned," International J. Applied Glass Science 1(2), 131-142 (2010)

17. J. E. Goell and R. D. Standley, "Integrated optical circuits," Proc. IEEE 58(10), 1504-1512(1970).

18. D. Gloge, "Optical waveguide transmission," Proc. IEEE 58(10), $1513-1522(1970)$

19. A. D. Pearson, W. G. French, and E. G. Rawson, "Preparation of a light focusing glass rod by ion-exchange techniques," Appl. Phys. Lett. 15(2), 76-77 (1969).

20. D. T. Moore, "Gradient-index optics: a review," Appl. Opt. 19(7), $1035-1038$ (1980).

21. T. Izawa and H. Nakagome, "Optical waveguide formed by electrically induced migration of ions in glass plates," Appl. Phys. Lett. 21(12), 584-586 (1972)

22. T. G. Giallorenzi, E. J. West, R. Kirk, R. Ginther, and R. A. Andrews, "Optical waveguides formed by thermal migration of ions in glass," Appl. Opt. 12(6), 1240-1245 (1973).

23. M. Saruwatari and T. Izawa, "Nd-glass laser with three-dimensional optical waveguide," Appl. Phys. Lett. 24(12), 603-605 (1974).

24. A. Gedeon and B. Carnstam, "Radiation loss in bends of diffused optical stripe guides," Opt. Quantum Electron. 7(6), 456-458 (1975).

25. J. G. Gallagher and R. M. de la Rue, "Single-mode stripe optical waveguides formed by silver ion exchange," Electron. Lett. 12(16), 397-398 (1976).

26. G. H. Chartier, P. Jaussaud, A. D. de Oliveira, and O. Parriaux, "Fast fabrication method for thick and highly multimode optical waveguides," Electron. Lett. 13(25), 763-764 (1977).

27. G. H. Chartier, P. Jaussaud, A. D. de Oliveira, and O. Parriaux, "Optical waveguides fabricated by electric-field controlled ion exchange in glass," Electron. Lett. 14(5), 132-134 (1978). 
28. J. Viljanen and M. Leppihalme, "Fabrication of optical strip waveguides with nearly circular cross section by silver ion migration technique," J. Appl. Phys. 51(7), 3563-3565 (1980).

29. H.-J. Lilienhof, E. Voges, D. Ritter, and B. Pantschev, "Field-induced index profiles of multimode ion-exchanged strip waveguides," IEEE J. Quantum Electron. QE-18(11), 1877-1883 (1982).

30. V. Neuman, O. Parriaux, and L. M. Walpita, "Double-alkali effect: influence of index profile of ion-exchanged waveguides," Electron. Lett. 15(22), 704-706 1979.

31. G. Stewart, C. A. Millar, P. J. R. Laybourn, C. D. W. Wilkinson, and R. M. de la Rue, "Planar optical waveguides formed by silver ion migration in glass," IEEE J. Quantum Electron. QE-13(4), 192-200 (1977).

32. C. A. Millar and R. H. Hutchins, "Manufacturing tolerances for silversodium ion-exchanged planar optical waveguides," J. Phys. D: Appl. Phys. 11(11), 1567-1576 (1978).

33. G. Stewart and P. Laybourn, "Fabrication of ion-exchanged optical waveguides from dilute silver nitrate melts," IEEE J. Quantum Electron. QE-14(12), 930-934 (1978).

34. I. Savatinova, E. Nadjakov, and L. Masher, "Determination of refractive index profiles in diffused optical waveguides," Appl. Phys. 12(3), 293-296 (1977).

35. V. Hinkov and W. Sohler, "Reduced depth polarizer for integrated optics," Appl. Phys. 14, 229-230 (1977).

36. J. C. Campbell, "Tapered waveguides for guided wave optics," Appl. Opt. 18(6), 900-902 (1979).

37. T. Suhara, J. Viljanen, and M. Leppihalme, "Integrated-optic wavelength multi- and demultiplexers using a chirped grating and an ionexchanged waveguide," Appl. Opt. 21(12), 2196-2199 (1982).

38. T. Findakly and B. Chen, "Single-mode integrated optical 1 X N star coupler," Appl. Phys. Lett. 40(7), 549-550 (1982).

39. M. Haruna and J. Koyama, "Thermooptic deflection and switching in glass," Appl. Opt. 21(19), 3461-3465 (1982).

40. R. G. Walker, C. D. W. Wilkinson, and J. A. H. Wilkinson, "Integrated optical waveguiding structures made by silver ion-exchange in glass. 1: The propagation characteristics of stripe ion-exchanged waveguides; a theoretical and experimental investigation," Appl. Opt. 22(12), 19231928 (1983)

41. R. G. Walker and C. D. W. Wilkinson, "Integrated optical waveguiding structures made by silver ion-exchange in glass. 2: directional coupler and bends," Appl. Opt. 22(12), 1929-1936 (1983).

42. R. G. Walker and C. D. W. Wilkinson, "Integrated optical ring resonators made by silver ion-exchange in glass," Appl. Opt. 22(7), 1029_ 1035 (1983)

43. T. J. Cullen and C. D. W. Wilkinson, "Radiation losses from singlemode optical Y junctions formed by silver-ion exchange in glass," Opt. Lett. 10(4), 134-136 (1984).

44. G. L. Yip and J. Finak, "Directional-coupler power divider by two-step K+-ion exchange," Opt. Lett. 9(9), 423-425 (1984).

45. B. Pantchev, "Multimode strip waveguide formed by ion-electrodiffusion from solid state silver: Side diffusion reduction," Opt. Commun. 60(6), 373-375 (1986).

46. B. G. Pantchev, "One-step field-assisted ion exchange for fabrication of buried multimode optical strip waveguides," Electron. Lett. 23(22), 1188-1190 (1987)

47. M. Seki, H. Hashizume, and R. Sugawara, "Two-step purely thermal ion-exchange technique for single-mode waveguide devices in glass," Electron. Lett. 24(20), 1258-1259 (1988).

48. J. L. Jackel, "Glass waveguides made using low melting point nitrate mixtures," Appl. Opt. 27(3), 472-475 (1988)

49. H. S. Monteiro, J. C. Said, J. F. Mendes, and R. Srivastava, "An apparatus for fabrication of optical waveguides by electromigration," Rev. Sci. Instrum. 68(11), 4014-4016 (1997).

50. M. N. Weiss and R. Srivastava, "Determination of ion-exchanged channel waveguide profile parameters by mode-index measurements," Appl. Opt. 34(3), 455-458 (1995).

51. B. Pantchev, P. Danesh, and Z. Nikolov, "Field-assisted ion exchange in glass: the effect of masking films," Appl. Phys. Lett. 62(11), 12121214 (1993).

52. B. Pantchev and P. Danesh, "Masking problem in the fabrication of optical waveguide structures in glass by double ion exchange," Jpn. J. Appl. Phys. 36(7A), 4320-4322 (1997).

53. D. Cheng, J. Saarinen, H. Saarikoski, and A. Tervonen, "Simulation of field-assisted ion exchange for glass channel waveguide fabrication: effect of nonhomogeneous time-dependent electric conductivity," Opt. Commun. 137(4-6), 233-238 (1997)

54. P. Madasamy, B. R. West, M. M. Morrell, D. F. Geraghty, S. Honkanen, and N. Peyghambarian, "Buried ion-exchanged glass waveguides: burial depth dependence on the waveguide width," Opt. Lett. 28(3), 1132-1134 (2003).

55. X. Prieto Blanco, "Interferometric characterization and analysis of silver-exchanged glass waveguides buried by electromigration: slab, channel and slab-sided channel configurations," J. Opt. A: Pure Appl. Opt. 8(2), 123-133 (2006)

56. A. Beguin, T. Dumas, M. J. Hackert, R. Jansen, and C. Nissim, "Fabrication and performance of low loss optical components made by ion exchange in glass," J. Lightwave Technol. 6(10), 1483-1487 (1988)

57. M. McCourt, "Status of glass and silicon-based technologies for passive components," Eur. Trans.Telecommun. 4(6), 685-689 (1993).

58. L. Ross, H. Lilienhof, U. Hölscher, H. F. Schlaak, and A. Brandenburg, "Improved substrate glass for planar waveguides by Csion exchange," in Technical Digest, Topical Meeting on Integrated and Guided-Wave Optics, pp. 25-26, Optical Society of America, Washington, DC (1986)

59. J. Albert and G. L. Yip, "Stress-induced index change for $\mathrm{K}+-\mathrm{Na}+$ ion exchange in glass," Electron. Lett. 23(14), 737-738 (1987).

60. A. Brandenburg, "Stress in ion-exchanged glass waveguides," J. Lightwave Technol. 4(10), 1580- 1593 (1986).

61. R. Araujo, "Colorless glasses containing ion-exchanged silver," Appl. Opt. 31(25), 5221-5224 (1992).

62. N. Fabricius, H. Öeste, H.-J. Guttmann, H. Quast, and L. Ross, "BGG 31: a new glass for multimode waveguide fabrication," in Proceeding of EFOC/LAN 88, pp. 59-62, Information Gatekeepers, Boston, MA (1988).

63. S. Honkanen, "Silver-film ion-exchange technique," in Introduction to Glass Integrated Optics, S. I. Najafi, Ed., pp. 39-71, Artech House, Norwood, MA (1992).

64. D. Kapila and J. L. Plawsky, "Diffusion processes for integrated waveguide fabrication in glasses: a solid-state electrochemical approach," Chem. Eng. Sci. 50(16), 2589-2600 (1995).

65. H. C. Zhenguang, R. Srivastava, and R. V. Ramaswamy, "Single-mode buried channel waveguide by single-step electromigration technique using silver film," Appl. Phys. Lett. 53(18), 1681-1683 (1988).

66. A. Tervonen, P. Pöyhönen, S. Honkanen, M. Tahkokorpi, and S. Tammela, "Examination of two-step fabrication methods for single-mode fiber compatible ion-exchanged waveguides," Appl. Opt. 30(3), 338343 (1991).

67. A. Tervonen, P. Pöyhönen, S. Honkanen, and M. Tahkokorpi, "A guided-wave Mach-Zehnder interferometer structure for wavelength multiplexing," IEEE Photonics Technol. Lett. 3(6), 516-518 (1991).

68. P. Pöyhönen, S. Honkanen, A. Tervonen, M. Tahkokorpi, and J. Albert, "Planar 1/8 splitter in glass by photoresist masked silver film ion exchange," Electron. Lett. 27(15), 1319-1320 (1991).

69. G. H. Chartier, P. J. R. Laybourn, and A. Girod, "Masking process for double-ion-exchanged glass optical waveguides," Electron. Lett. 22(17), 925-926 (1986).

70. A. Tervonen and S. Honkanen, "Model for waveguide fabrication in glass by two-step ion exchange with ionic masking," Opt. Lett. 13(1), 71-73 (1988).

71. G. C. Righini and G. Molesini, "Design of optical-waveguide homogeneous refracting lenses," Appl. Opt. 27(20), 4193-4199 (1988).

72. J. Saarinen, S. Honkanen, S. I. Najafi, and J. Huttunen, "Double-ionexchange process in glass for the fabrication of computer-generated waveguide holograms," Appl. Opt. 33(16), 3353-3359 (1994).

73. S. Honkanen, P. Pöyhönen, A. Tervonen, and S. I. Najafi, "Waveguide coupler for potassium- and silver-ion-exchanged waveguides in glass," Appl. Opt. 32(12), 2109-2111 (1993).

74. W. J. Wang, S. Honkanen, S. I. Najafi, and A. Tervonen, "Loss characteristics of potassium and silver double-ion-exchanged glass waveguides," J. Appl. Phys. 74(3), 1529-1533 (1993).

75. P. K. Tien and R. Ulrich, "Theory of prism-film coupler and thin film light guides," J. Opt. Soc. Am. 60(10), 1325-1337 (1970).

76. J. M. White and P. F. Heidrich, "Optical waveguide refractive index profiles determined from measurement of mode indices: a simple analysis," Appl. Opt. 15(1), 151-155 (1976).

77. K. S. Chiang, "Construction of refractive-index profiles of planar dielectric waveguides from distribution of effective indexes," J. Lightwave Technol. LT-3(2), 385-391 (1985).

78. S. Pelli, G. C. Righini, A. Scaglione, G.-L. Yip, P. Noutsious, A. Bräuer, P. Dannberg, J. Linares, C. G. Reino, G. Mazzi, F. Gonella, R. Rimet, and I. Schanen, "Testing of optical waveguides (TOW) cooperative project: preliminary results of the characterisation of Kexchanged waveguides," Proc. SPIE 2212, 126-131 (1994).

79. J. Viljanen and J. Kurki, "Planar waveguide refractive-index profiling using modal intensity distributions," Appl. Opt. 21(23), 4321-4323 (1982).

80. R. Göring and M. Rothhardt, "Application of the refracted near-field technique to multimode planar and channel waveguides in glass," J. Opt. Commun. 7(3), 82-85 (1986).

81. P. Mrozek, E. Mrozek, and T. Lukaszewicz "Determination of refractive index profiles of $\mathrm{Ag}+-\mathrm{Na}+$ ion-exchange multimode strip waveguides by variable wavefront shear double-refracting interferometry microinterferometry," Appl. Opt. 45(4), 756-763 (2006).

82. B. Pantchev and Z. Nikolov, "Characterization of refractive index profiles in silver-sodium ion-exchanged glass waveguides for homogeneous refracting waveguide structures," IEEE J. Quantum Electronics 29(9), 2459-2465 (1993).

83. G. Li, K. A. Winick, H. C. Griffin, and J. S. Hayden, "Systematic modeling study of channel waveguide fabrication by thermal silver ion exchange," Appl. Opt. 45(8), 1743-1755 (2006). 
84. A. Lupascu, A. Kevorkian, T. Boudet, F. Saint-André, D. Persegol, and M. Levy, "Modeling ion exchange in glass with concentrationdependent diffusion coefficients and mobilities," Opt. Eng. 35(6), 1603-1610 (1996).

85. P. Äyräs, G. N. Conti, S. Honkanen, and N. Peyghambarian, "Birefringence control for ion-exchanged channel glass waveguides," Appl. Opt. 37(36), 8400-8405 (1998).

86. A. Tervonen, "Theoretical analysis of ion-exchanged glass waveguides," in Introduction to Glass Integrated Optics, S. I. Najafi, Ed., pp. 73-105, Artech House, Norwood, MA (1992).

87. H. Kahnt, "Ionic transport in glasses," J. Non-Cryst. Solids 203, 225231 (1996).

88. P. Maass, "Toward a theory for the mixed alkali effect in glasses," J. Non-Cryst. Solids 255(1), 35-46 (1999).

89. S. A. Poling and S. N. Houde-Walter, "Relaxation behaviour of silversodium interdiffusion in a boroaluminosilicate glass," J. Non-Cryst. Solids 272(1), 39-45 (2000)

90. X. Prieto-Blanco, "Electro-diffusion equations of monovalent cations in glass under charge neutrality approximation for optical waveguide fabrication," Opt. Mat. 31(2), 418-428 (2008).

91. O. L. Anderson and D. A. Stuart, "Calculation of activation energy of ionic conductivity in silica glasses by classical methods," J. Am. Cer Soc. 37(12), 573-580 (1954).

92. R. Kirchheim and D. Paulmann, "The relevance of site energy distribution for the mixed alkali effect," J. Non-Cryst. Solids 286(3), 210-223 (2001)

93. A. Quaranta, E. Cattaruzza, and F. Gonella, "Modelling the ion exchange process in glass: phenomenological approaches and perspectives," Mat. Sci. Eng. B 149(2), 133-139 (2008).

94. R.-P. Salmio, J. Saarinen, J. Turunen, and A. Tervonen, "Graded-index diffractive elements by thermal ion exchange in glass," Appl. Phys. Lett. 66(8), 917-919 (1995).

95. R.-P. Salmio, H. Saarikoski, J. Saarinen, J. Westerholm, and J. Turunen, "Three-dimensionally modulated graded-index diffractive elements by thermal ion exchange in glass," Opt. Lett. 22(9), 591-593 (1997).

96. G. L. Yip, P. C. Noutsios, and K. Kishioka, "Characteristics of optical waveguides made by electric-field-assisted $\mathrm{K}^{+}$-ion exchange," Opt. Lett. 15(14), 789-791 (1990).

97. P. J. Masalkar, "Calculation of concentration profile in ion-exchange waveguides by finite difference ADI method," Optik 95(4), 168-172 (1994)

98. B. R. West, P. Madasamy, N. Peyghambarian, and S. Honkanen, "Modeling of ion-exchanged glass waveguide structures," J. Non-Cryst. Solids 347(1-3), 18-26 (2004)

99. D. W. Peaceman and H. H. Rachford, Jr., "The numerical solution of parabolic and elliptic differential equations," J. Soc. Ind. Appl. Math. 3, 28-41 (1955).

100. H. Saarikoski, R. P. Salmio, J. Saarinen, T. Eirola, and A. Tervonen, "Fast numerical solution of nonlinear diffusion equation for the simulation of ion-exchanged micro-optics components in glass," Opt. Commun. 134(1-6), 362-370 (1997).

101. P. Chludzinski, R. V. Ramaswamy, and T. J. Anderson, "Ion exchange between soda-lime silica glass and sodium nitrate-silver nitrate molten salts," Phys. Chem. Glasses 28(5), 169-173 (1987).

102. H. M. Garfinkel, "Ion-exchange equilibria between glass and molten salts," J. Phys. Chem. 72(12), 4175-4181 (1968).

103. S. D. Fantone, "Refractive index and spectral models for gradientindex materials," Appl. Opt. 22(3), 432-440 (1983).

104. R. J. Rao, Structural Chemistry of Glasses, Elsevier, New York (2002).

105. A. Opilski, R. Rogoziński, M. Błahut, P. Karasiński, K. Gut, and Z. Opilski, "Technology of ion exchange in glass and its application in waveguide planar sensors," Opt. Eng. 36(6), 1625-1638 (1997).

106. P. Y. Choo, J. A. Frantz, J. T. A. Carriere, D. L. Mathine, R. K. Kostuk, and N. Peyghambarian, "Measurement and modeling of ionexchange parameters for IOG-10 glass," Opt. Eng. 42(10), 2812-2816 (2003).

107. K. S. Chiang "Effective-index function method for the analysis an design of inhomogeneous planar waveguides based on the WKB equation," Opt. Commun. 84(5-6), 258-263 (1991).

108. B. R. West and S. Honkanen, "Determination of ion exchange parameters by a genetic algorithm," Opt. Eng. 44(9), 094602 (2005).

109. P. Benech, D. Persegol, and F. Saint Andre, "A glass ion exchanged Mach-Zehnder interferometer to stabilize the frequency of a laser diode," J. Phys. D 23(5), 617-619 (1990).

110. S. Das, D. Geraghty, S. Honkanen, and N. Peyghambarian, "MMI splitters by ion-exchange in glass," Proc. SPIE 3936, 239-247 (2000).

111. D. Kasprzak, M. Blahut, and E. Maciak, "Applications of multimode interference effects in gradient waveguides produced by ion-exchange in glass," Eur. Phys. J. Special Topics 154(1), 113-116 (2008).

112. B. Buchold, C. Glingener, D. Culemann, and E. Voges "Polarization Insensitive Ion-Exchanged Arrayed-Waveguide Grating Multiplexers in Glass," Fiber Integr Optics, 17(4), 279-298 (1998).
113. A. Tervonen, S. Honkanen, and S. I. Najafi, "Analysis of symmetric directional couplers and asymmetric Mach-Zehnder interferometers as $1.30 / 1.55 \mu \mathrm{m}$ dual wavelength demultiplexers/ multiplexers," Opt. Eng. 32(9), 2083-2091 (1993).

114. B. West and S. Honkanen, "MMI devices with weak guiding designed in three dimensions using a genetic algorithm," Opt. Express 12(12), 2716-2722 (2004)

115. B. R. West and D. V. Plant, "Optimization of non-ideal multimode interference devices," Opt. Comm. 279, $72-78$ (2007).

116. S. Ruschin, G. Hurwitz, T. Hurwitz, A. Kepten, E. Arad, Y. Soreq, and S. Eckhouse, "Glass ion-exchange technology for wavelength management applications," Proc. SPIE 4944, 150-158 (2003).

117. T. Hurvitz, S. Ruschin, D. Brooks, G. Hurvitz, and E. Arad, "Variable optical attenuator based on ion-exchange technology in glass," J. Lightwave Technol. 23(5), 1918-1922 (2005).

118. J. E. Roman and K. A. Winick, "Photowritten gratings in ionexchanged glass waveguides," Opt. Lett. 18(10), 808-810 (1993).

119. C. Montero, C. Gomez-Reino, and J. L. Brebner, "Planar Bragg gratings made by excimer-laser modification of ion-exchanged waveguides," Opt. Lett. 24(21), 1487-1489 (1999).

120. D. F. Geraghty, D. Provenzano, W. K. Marshall, S. Honkanen, A. Yariv, and N. Peyghambarian, "Gratings photowritten in ion-exchanged glass channel waveguides," Electron. Lett. 35(7), 585-587 (1999).

121. J. M. Castro, D. F. Geraghty, B. R. West, and S. Honkanen, "Fabrication and Comprehensive Modeling of Ion-Exchanged Bragg Optical Add-Drop Multiplexers," Appl. Opt. 43(33), 6166-6173 (2004)

122. D. Bucci, J. Grelin, E. Ghibaudo, and J.-E. Broquin, "Realization of a 980-nm/1550-nm pump-signal (de)multiplexer made by ion-exchange on glass using a segmented asymmetric Y-Junction," IEEE Photonics Technol. Lett. 19(9), 698-700 (2007).

123. G. Zhang, S. Honkanen, A. Tervonen, C.-M. Wu, and S. I. Najafi, "Glass integrated optics circuit for 1.48/1.55- and 1.30/1.55- $\mu$ m wavelength division multiplexing and 1/8 splitting," Appl. Opt. 33(16), 3371-3374 (1994)

124. J. Grelin, E. Ghibaudo, and J.-E. Broquin, "Study of deeply buried waveguides: A way towards 3D integration," Mat. Sci. Eng.: B 149(2, 185-189 (2008)

125. R. I. MacDonald, D. K. W. Lam, and B. A. Syrett, "Hybrid optoelectronic integrated circuit," Appl. Opt. 26(5), 842-844 (1987).

126. D. R. Larson and R. J. Phelan, Jr., "Fast optical detector deposited on dielectric channel waveguides," Opt. Eng. 27(6), 503-506 (1988).

127. D. R. Larson and R. J. Phelan, Jr., "Hydrogenated amorphous germanium detectors deposited onto channel waveguides," Opt. Lett. 15(10), 544-546 (1990)

128. A. Yi-Yan, W. K. Chan, T. J. Gmitter, L. T. Florez, J. L. Jackel, E. Yablonovitch, R. Bhat, and J. P. Harbison, "Grafted GaAs detectors on lithium niobate and glass optical waveguides," IEEE Photonics Technol. Lett. 1(11), 379-380 (1989).

129. T. J. Cullen, C. N. Ironside, C. T. Seaton, and G. I. Stegeman, "Semiconductor-doped glass ion-exchanged waveguides," Appl. Phys. Lett. 49(21), 1403-1405 (1986).

130. A. Gabel, K. W. DeLong, C. T. Seaton, and G. I. Stegeman, "Efficient degenerate four-wave mixing in an ion-exchanged semiconductordoped glass waveguide," Appl. Phys. Lett. 51(21), 1682-1684 (1987).

131. N. Finlayson, W. C. Banyai, E. M. Wright, C. T. Seaton, and G. I. Stegeman, "Picosecond switching induced by saturable absorption in a nonlinear directional coupler," Appl. Phys. Lett. 53(13), 1144-1146 (1988).

132. J. M. Auxier, S. Honkanen, A. Schülzgen, M. M. Morrell, M. A. Leigh, S. Sen, N. F. Borrelli, and N. Peyghambarian, "Silver and potassium ion-exchanged waveguides in glasses doped with $\mathrm{PbS}$ semiconductor quantum dots," J. Opt. Soc. Am. B 23, 1037-1045 (2006).

133. J. S. Aitchison, A. M. Weiner, Y. Silberberg, M. K. Oliver, J. L. Jackel, D. E. Leaird, E. M. Vogel, and P. W. E. Smith, "Observation of spatial optical solitons in a nonlinear glass waveguide," Opt. Lett. 15(9), 471473 (1990).

134. J. S. Aitchison, A. M. Weiner, Y. Silberberg, D. E. Leaird, M. K. Oliver, J. L. Jackel, and P. W. E. Smith, "Experimental observation of spatial soliton interactions," Opt. Lett. 16(1), 15-17 (1991).

135. J. L. Jackel, E. M Vogel, and J. S. Aitchison, "Ion-exchanged optical waveguides for all-optical switching," Appl. Opt. 29(21), 3126-3129 (1990).

136. S. I. Najafi, W.-J. Wang, J. F. Currie, R. Leonelli, and J. L. Brebner, "Fabrication and characterization of neodymium-doped glass waveguides," IEEE Photonics Technol. Lett. 1(5), 109-110 (1989).

137. H. Aoki, E. Ishikawa, and Y. Asahara, "Nd3 + -doped glass waveguide amplifier at 1.054- $\mu \mathrm{m}$, " Electron. Lett. 27(25), 2351-2353 (1991).

138. N. A. Sanford, K. J. Malone, D. R. Larson, and R. K. Hickernell, "Y-branch waveguide glass laser and amplifier," Opt. Lett. 16(15), $1168-1170$ (1991)

139. A. N. Miliou, X. F. Cao, R. Srivastava, and R. V. Ramaswamy, " 15 dBamplification at $1.06 \mu \mathrm{m}$ in ion-exchanged silicate glass waveguides," IEEE Photon. Technol. Lett. 5(4), 416-418 (1993). 
140. J. E. Roman and K. A. Winick "Neodymium-doped glass channel waveguide laser containing an integrated distributed Bragg reflector," Appl. Phys. Lett. 61(23), 2744-2746 (1993).

141. N. A. Sanford, J. A. Aust, K. J. Malone, and D. R. Larson, "Linewidth narrowing in an imbalanced Y-branch waveguide laser," Opt. Lett. 18(4), 281-283 (1993).

142. J. A. Aust, K. J. Malone, D. L. Veasey, N. A. Sanford, and A. Roshko, "Passively Q-switched Nd-doped waveguide laser," Opt. Lett. 19(22) 1849-1851 (1994)

143. W. J. Miniscalco, "Erbium-doped glasses for fiber amplifiers at 1500 nm," J. Lightwave Technol. 9(2), 234- 250 (1991).

144. D. Barbier, J. M. Delavaux, A. Kevorkian, P. Gastaldo, and J. M. Jouanno, "Yb/Er integrated optics amplifiers on phosphate glass in single and double pass configurations," in Proc. Optical Fiber Communication (OFC'95), postdeadline paper PD-3, San Diego, CA, February 26-March 3, 1995.

145. D. L. Veasey, D. S. Funk, N. A. Sanford, and J. Hayden, "Arrays of distributed-Bragg-reflector waveguide lasers at $1536 \mathrm{~nm}$ in $\mathrm{Yb} / \mathrm{Er}$ codoped phosphate glass," Appl. Phys. Lett. 74(6), 789-791 (1999).

146. S. Blaize, L. Bastard, C. Cassagnètes, and J. E. Broquin, "Multiwavelengths DFB waveguide laser arrays in $\mathrm{Yb}-\mathrm{Er}$ codoped phosphate glass substrate," IEEE Photon. Technol. Lett. 15(4), 516-518 (2003).

147. S. Honkanen, S. I. Najafi, P. Pöyhönen, G. Orcel, W. J. Wang, and J. Chrostowski, "Silver-film ion-exchanged single-mode waveguides in Er-doped phosphate glass," Electron. Lett. 27(23), 2167-2168 (1991).

148. F. D. Patel, S. DiCarolis, P. Lum, S. Venkatesh, and J. N. Miller, "A compact high-performance optical waveguide amplifier," IEEE Photon. Technol. Lett. 16(12), 2607-2609 (2004).

149. D. Barbier, M. Rattay, F. Saint Andŕe, G. Clauss, M. Trouillon, A. Kevorkian, J.-M. P. Delavaux, and E. Murphy, "Amplifying fourwavelength combiner, based on Erbium/Ytterbium-doped waveguide amplifiers and integrated splitters," IEEE Photon. Techol. Lett. 9(3), 315-317 (1997)

150. K. C. Reichmann, P. P. Iannone, M. Birk, N. J. Frigo, D. Barbier, C. Cassagnettes, T. Garret, A. Verlucco, S. Perrier, and J. Philipsen, "An eight-wavelength $160-\mathrm{km}$ transparent metro WDM ring network featuring cascaded erbium-doped waveguide amplifiers," IEEE Photon. Technol. Lett. 13(10), 1130-1132 (2001).

151. http://www.teemphotonics.com/, accessed Jan. 20, 2011.

152. T. Feuchter, E. K. Mwarania, J. Wang, L. Reekie, and J. S. Wilkinson, "Erbium-doped ion-exchanged waveguide lasers in BK-7 glass," IEEE Photon. Technol. Lett. 4(6), 542-544 (1992).

153. P. P. Madasamy, G. Nunzi Conti, P. Pöyhönen, M. M. Morrell, D. F. Geraghty, S. Honkanen, and N. Peyghambarian, "Waveguide distributed Bragg reflector laser arrays in erbium doped glass made by Ag film ion exchange," Opt. Eng. 41(5), 1084-1086 (2002).

154. L. Bastard, S. Blaize, and J.-E. Broquin, "Glass integrated optics ultra narrow linewidth DFB lasers for DWDM applications," Opt. Eng. 42(10), 2800-2804 (2003)

155. S. Pissadakis, A. Ikiades, P. Hua, A. K. Sheridan, and J. S. Wilkinson, "Photosensitivity of ion-exchanged Er-doped phosphate glass using $248 \mathrm{~nm}$ excimer laser radiation," Opt. Express 12(14), 3131-3136 (2004).

156. S. Yliniemi, S. Honkanen, A. Ianoul, A. Laronche, and J. Albert, "Photosensitivity and volume gratings in phosphate glasses for rareearth-doped ion-exchanged optical waveguide lasers," J. Opt. Soc. Am. $B$ 23(12), 2470-2478 (2006)

157. S. Yliniemi, J. Albert, Q. Wang, and S. Honkanen, "UV-exposed Bragg gratings for laser applications in silver-sodium ion-exchanged phosphate glass waveguides," Opt. Express 14(7), 2898-2903 (2006).

158. S. D. Gonzone, J. S. Hayden, D. S. Funk, A. Roshko, and D. L. Veasey, "Hybrid glass substrates for waveguide device manufacture," Opt. Lett. 26(8), 509-511 (2001).

159. H. Schröder, N. Arndt-Staufenbiel, M. Cygon, and W. Scheel, "Planar glass waveguides for high performance electrical-optical-circuitboards (EOCB)-the glass-layer-concept-," in Proc. 53rd Electronic Components and Technology Conf., pp. 1053-1059, IEEE (2003)

160. H. Schröder, N. Arndt-Staufenbiel, L. Brusberg, and T. Tekin, "Optical coupling using thin glass based arrayed waveguide components," in Technical Digest of the Fourteenth Microoptics Conference, H. Thienpont, Ed., pp. 56-57, Microoptics Group, The Optical Society of Japan, Tokyo (2008).

161. H. Schröder, L. Brusberg, and N. Arndt-Staufenbiel, "New options for chip-to-chip photonic packaging by using thin glass based waveguide substrates on board and module level," Proc. SPIE 7607, 76070F (2010).

162. H. Schröder, N. Arndt-Staufenbiel, A. Beier, F. Ebling, M. Franke, E. Griese, S. Intemann, J. Kostelnik, T. Kühler, R. Mödinger, I. Roda, and I. Schlosser, "Thin glass based electrical-optical circuit boards (EOCB) using ion-exchange technology for graded-index multimode waveguides;" in Proc. 58th Electronic Components and Technology Conf., pp. 268-275, IEEE (2008)

163. L. Ross, "Ion-exchanged glass waveguide sensors," SPIE Critical Reviews of Optical Science and Technology CR53, 180-199 (1994).
164. O. M. Parriaux, P. Roth, and G. Voirin, "Multimode glass integrated optics," SPIE Critical Reviews of Optical Science and Technology CR53, 295-320 (1994).

165. P. V. Lambeck, "Integrated optical sensors for the chemical domain," Meas. Sci. Technol. 17(8), R93-R116 (2006).

166. A. Yimit, A. G. Rossberg, T. Amemiya, and K. Itoh, "Thin film composite optical waveguides for sensor applications: a review," Talanta 65(5), 1102-1109 (2005)

167. J. Dostalek, J. Ctyroky, J. Homola, E. Brynda, M. Skalsky, P. Nekvindova, J. Spirkova, J. Skvor, and J. Schrofel, "Surface plasmon resonance biosensor based on integrated optical waveguide," Sens. Actuators B 76(1-3), 8-12 (2001).

168. R. Mazurczyk, J. Vieillard, A. Bouchard, B. Hannes, and S. Krawczyk, "A novel concept of the integrated fluorescence detection system and its application in a lab-on-a-chip microdevice," Sens. Actuators $B$ 118(1-2), 11-19 (2006).

169. J. Vieillard, R. Mazurczyk, L.-L. Boum, A. Bouchard, Y. Chevolot, P. Cremillieu, B. Hannes, and S. Krawczyk, "Integrated microfluidicmicrooptical systems fabricated by dry etching of soda-lime glass," Microelectron. Eng. 85(2), 465-469 (2008).

170. F. Malbet, P. Kern, I. Schanen-Duport, J.-P. Berger, K. RousseletPerraut, and P. Benech, "Integrated optics for astronomical interferometry-I. Concept and astronomical applications," Astron. Astrophys. Suppl. Ser. 138(1), 135-145 (1999).

171. J. P. Berger, K. Rousselet-Perraut, P. Kern, F. Malbet, I. SchanenDuport, F. Reynaud, P. Haguenauer, and P. Benech, "Integrated optics for astronomical interferometry. II. first laboratory whitelight interferograms," Astron. Astrophys. Suppl. Ser. 139(1), 173-177 (1999).

172. P. Haguenauer, J.-P. Berger, K. Rousselet-Perraut, P. Kern, F. Malbet I. Schanen-Duport, and P. Benech, "Integrated optics for astronomical interferometry. III. optical validation of a planar optics two-telescope beam combiner," Appl. Opt. 39(13), 2130-2139 (2000)

173. J. P Berger, P. Haguenauer, P. Kern, K. Perraut, F. Malbet, I. Schanen, M. Severi, R. Millan-Gabet, and W. Traub, "Integrated optics for astronomical interferometry IV. first measurements of stars," Astron. Astrophys., 376(3), L31-L34 (2001).

174. E. Laurent, K. Rousselet-Perraut, P. Benech, J. P. Berger, S. Gluck, P. Haguenauer, P. Kern, F. Malbet, and I. Schanen-Duport, "Integrated optics for astronomical interferometry-V. extension to the K band," Astron. Astrophys. 390(3), 1171-1176 (2002).

175. L. Labadie and O. Wallner, "Mid-infrared guided optics: a perspective for astronomical instruments," Opt. Express 17(3), 1947-1962 (2009)

176. T. Luo, S. Jiang, G. Nunzi Conti, S. Honkanen, S. B. Mendes, and N. Peyghambarian, "Ag $+/ \mathrm{Na}+$ exchanged channel waveguides in germanate glass," Electron. Lett. 34(23), 2239-2240 (1998)

177. J. Grelin, A. Bouchard, E. Ghibaudo, and J. E. Broquin, "Study of $\mathrm{Ag}+/ \mathrm{Na}+$ ion-exchange diffusion on germanate glasses: realization of single-mode waveguides at the wavelength of $1.55 \mu \mathrm{m}$," Mater. Sci. Eng. B 149(2), 190-194 (2008).

178. K. Grujic, O. G. Helles $\varnothing$, J. S. Wilkinson, and J. P. Hole, "Optical propulsion of microspheres along a channel waveguide produced by Cs + ion-exchange in glass," Opt. Comm. 239(4-6), 227-235 (2004)

179. G. Lerondel, A. Sinno, L. Chassagne, S. Blaize, P. Ruaux, A. Bruyant, S. Topcu, P. Royer, and Y. Alayli, "Enlarged near-field optical imaging," J. Appl. Phys. 106(4), 044913-1-4 (2009).

180. A. Hassanzadeh, M. Nitsche, S. Armstrong, N. Nabavi, R. Harrison S. J. Dixon, U. Langbein, and S. Mittler, "Optical waveguides formed by silver ion exchange in Schott SG11 glass for waveguide evanescent field fluorescence microscopy: evanescent images of HEK293 cells," J. Biomed. Opt. 15(3), 036018-1-6 (2010).

181. P. Madasamy, S. Honkanen, D. F. Geraghty, and N. Peyghambarian, "Single-mode tapered waveguide laser in Er-doped glass with multimode-diode pumping," Appl. Phys. Lett. 82, 1332-1334 (2003).

182. H.-K. Hsiao and K. A. Winick, "Planar glass waveguide ring resonators with gain," Opt. Express 15(26), 17783-17797 (2007).

183. M. A. Khalil, G. Vitrant, P. Raimond, P. A. Chollet, and F. Kajzar, "Modal dispersion phase-matched frequency doubling in composite planar waveguide using ion-exchanged glass and optically nonlinear poled polymer," Opt. Coтmun. 170(4-6), 281-284 (1999).

184. M. A. Khalil, G. Vitrant, P. Raimond, P. A. Chollet, and F. Kajzar, "Optical parametric amplification in composite polymer/ion exchanged planar waveguide," Appl. Phys. Lett. 77(23), 3713-3715 (2000).

185. A. Rodriguez, G. Vitrant, P. A. Chollet, and F. Kajzar, "Optical control of an integrated interferometer using a photochromic polymer," Appl. Phys. Lett. 79(4), 461-463 (2001).

186. I. E. Araci, R. Himmelhuber, C. T. DeRose, J. D. Luo, A. K.-Y. Jen, R. A. Norwood, and N. Peyghambarian, "Alignment-free fabrication of a hybrid electro-optic polymer/ion-exchange glass coplanar modulator," Opt. Express 18(20), 21038-21046 (2010). 


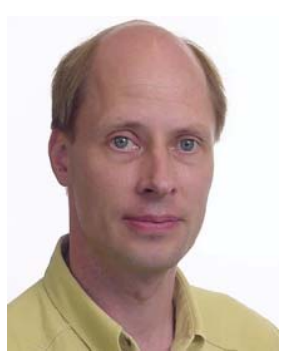

Ari Tervonen received his MS degree in physics in 1986, and, his $\mathrm{PhD}$ degree in physics in 1990, both from the University of Helsinki, Finland. He is currently senior researcher and docent in photonics at Aalto University, Espoo, Finland. Previously he has carried out integrated optics research at Technical Research Center of Finland, and has been principal scientist at Nokia Research Center in areas of optical communication and photonics for multimedia applications. He has also been involved with startup companies in integrated optics and optical communication. He has authored or coauthored over 100 journal and conference papers and 3 book chapters.

Brian R. West is an assistant professor in the Department of Physics and Computer Science, Wilfrid Laurier University, Waterloo, ON, Canada. He received his BEng degree in engineering physics from McMaster University, Hamilton, ON, Canada, in 1997, his MS degree from the Thayer School of Engineering, Dartmouth College, Hanover, NH, in 1999, and his MS and PhD degrees from the Optical Sciences Center, University of Arizona, Tucson, in 2002 and 2004 respectively, where his research included modeling the processing of ion-exchanged glass waveguides and devices. Subsequently, he was a postdoctoral research fellow in the Department of Electrical and Computer Engineering, University of Toronto, Toronto, ON, Canada, and then in the Department of Electrical and Computer Engineering McGill University, Montreal, QC, Canada. His current research interests include nonlinear plasmonic devices, Bragg reflection waveguides, and nonreciprocal waveguides. Prior to his academic career he was a research scientist with CoreTek, Inc., Burlington, MA, and MPB Communications, Pointe-Claire, QC, Canada, working on fiberbased devices. He is a Member of SPIE, OSA, and IEEE.

Seppo Honkanen received his $\mathrm{PhD}$ degree in electrical engineering in 1988 from Helsinki University of Technology. From 1989 to 1995 he was with Nokia Research Center as an R\&D manager of Integrated Optics and later a principal scientist in Optoelectronics. In 1995 he joined the Optical Sciences Center (OSC) at the University of Arizona, where he enjoyed the beauty of integrated optics in various faculty positions. During 1998 to 2001 he was vice president at NP Photonics, an Arizona based fiber laser company, he co-founded in 1998. In 2007 he became a Finland distinguished professor at Aalto University in Finland, and he is still an adjunct professor at the University of Arizona. His current research interests include silicon nanophotonics, active fibers, and silver nanoparticles in glass. 